\title{
Measuring Productivity and Efficiency of Traditional Chinese Medicine Hospitals from 2009 to 2016: A Bootstrap-Data Envelopment Analysis
}

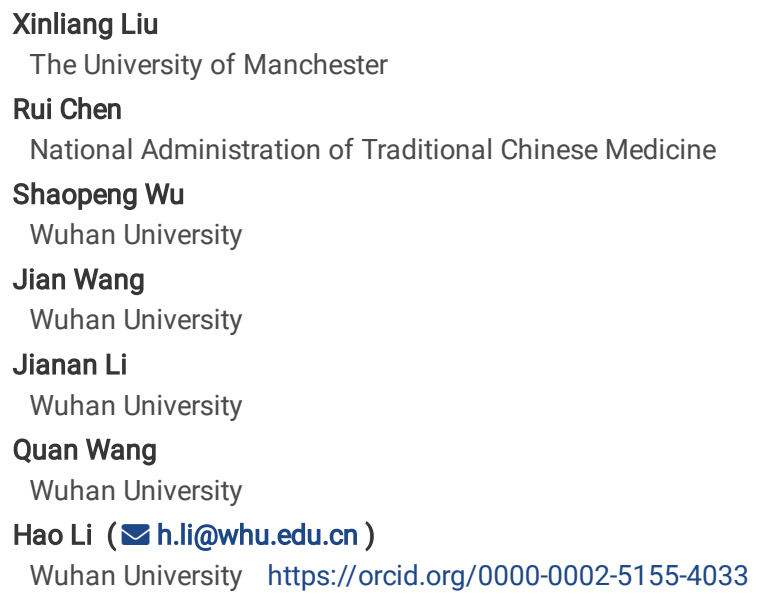




\section{Abstract}

Objective: It was to estimate the productivity and efficiency of Traditional Chinese Medicine (TCM) hospitals to provide empirical evidence for hospital managers and policy-makers to improve the management and quality of TCM service.

Methods: The data of the individual tertiary public TCM hospitals were collected from official Yearbooks of Traditional Chinese Medicine of China (20102017). Bootstrap-Malmquist-DEA was employed to measure the productivity and efficiency (2009-2016). SPSS23.0 was used to conduct the descriptive analysis of the input and output indicators. R3.2.1 was applied to calculate the productivity and efficiency with FEAR package. The statistical significance was set at $P<0.05$.

Results: The annual average growth rates of each indicator were $6.61 \%$ (health professionals), $8.15 \%$ (actual open beds), $7.08 \%$ (outpatients and inpatients) and $12.50 \%$ (discharged patients) respectively from 2009 to 2016 . Except the total factor productivity change (TFPC) between 2014 and 2015 , more than half of the TCM hospitals had TFPC scores over 1.000. The overall annual geo-mean TFPC score was 1.0379.

Conclusions: The overall annual rate of the TFPC of the tertiary public TCM hospitals was slightly increased. The technological progress was the main driver to improve the total factor productivity. The decreased technical efficiency was more affected by the decreased scale efficiency. The TCM hospitals need to pay attention to the development and innovation of the TCM technology, thereby improving the competitiveness. The TCM hospitals managers should pursuit the high quality, high efficiency and low cost of the TCM services.

\section{Introduction}

The Healthy China 2030 Planning Outline approved by China's Central Party Committee and the State Council has proposed that providing efficient and good quality of medical services and taking full advantage of Traditional Chinese Medicine (TCM) are two important goals (1). As an essential part of Chinese health care, the TCM development has been improving since the new medical reform due to a great number of policy documents highlighting TCM released by the Chinese government. As a result, the TCM services have become more accessible and more affordable, thereby decreasing the medical burden (2). For example, the outbreak of the new coronavirus pneumonia, named COVID-19 by the World Health Organization, has become a pandemic. Some traditional Chinese medicines, especially the Lung Cleansing and Detoxifying Decoction (Qing Fei Pai Du Tang), have shown therapeutic effects on mild and ordinary COVID-19 patients (3).

Traditional Chinese Medicine (TCM) hospitals are the dominant agents to provide the TCM services to the public in China (4). The TCM hospitals still face quite a number of challenges, like lower pricing of TCM outpatient services compared to their western medical counterparts (5), serious loss in human resources, and imbalanced structure of health professionals (6). What's worse, most of the TCM programs cannot be reimbursed by public health insurance schemes, which hampers patients from choosing TCM as their first choice(7). Further, how to provide the good quality of TCM services is also a big challenge for TCM hospitals. One of the key factors to provide the good quality of TCM services is to improve the operational efficiency of the TCM hospitals (8). However, the existing TCM resources are always limited. Therefore, how to improve the productivity and efficiency of TCM hospitals is quite pivotal for hospital managers and policy-makers to realize the optimal use of TCM resources.

Data Envelopment Analysis (DEA) has been widely applied to calculate the productivity and efficiency of healthcare institutions due to the characteristics of multiple inputs and multiple outputs (9). Furthermore, the Malmquist Productivity Index (MPI) model has been employed to indicate the dynamic productivity and efficiency changes of healthcare institutions or health systems $(10,11)$. There are several studies which adopted the MPI model to measure the productivity and efficiency of TCM hospitals $(4,8,12-14)$. However, there are some gaps on the current literature. None of the studies have focused on the individual tertiary public TCM hospitals at the national level. Besides, the MPI model adopted in current studies is classical. In 2007, Simar and Wilson proposed that Bootstrap could be combined with the classical MPI model to obtain the confidence intervals (Cls) of the productivity and efficiency scores, in order to eliminate the impact of the environmental and random factors on the estimation of productivity and efficiency scores $(15,16)$.

In our previous study, we applied the Bootstrap-DEA model to estimate the efficiency of other kinds of health institutions, like community health service centers (17), public general hospitals (18), and township hospitals (19). The purpose of this study was to measure the productivity and efficiency of the TCM hospitals with the Bootstrap-Malmquist-DEA model from 2009 to 2016 to provide empirical evidence for hospital managers and policy-makers to improve the management and quality of TCM medical services. It was the first time that our team conducted the estimation on the productivity and efficiency of TCM hospitals by employing Bootstrap-Malmquist-DEA.

\section{Methods}

\section{Input and output indicators selection}

According to a systematic review on the relative efficiency research of Chinese hospitals with DEA models conducted by Dong \& Li (9), they have suggested that the technical efficiency and allocative efficiency were always misunderstood by misuse of combination of monetary and volume indicators. The review and a previous study published by our research team (17) also have mentioned that price information of the indicators is much more difficult than the volume information to be accessed in Chinese hospitals. Therefore, based on the frequency of input and output indicators used most in current studies and the feasibility of data, in this study, the input indicators were the number of health professionals and the number of actual open beds, while the number of outpatients and inpatients and the number of discharged patients were selected as the output indicators.

\section{Research model}


The output-oriented Bootstrap-Malmquist-DEA model was employed to measure the productivity and efficiency of the individual tertiary public TCM hospitals. Basically, the total factor productivity changes (TFPC) and its decomposition index-technical efficiency changes (TEC) and technological changes (TC) can be obtained with the classical Malmquist-DEA model on the condition that all decision making units (DMUs)-TCM hospitals are operating in the frontier (20). Moreover, the technological changes can be decomposed into pure technical efficiency changes (PTEC) and scale efficiency changes (SEC). The basic formula is as follows:

TFPC $=$ TEC $\times T C=(P T E C \times S E C) \times T C$

Generally, when the productivity and efficiency scores are more than 1.0000, it means the rates are increasing; when the productivity and efficiency scores are equal to 1.0000 , it means the rates remain the same; and when the productivity and efficiency scores are less than 1.0000 , it means the rates are decreasing.

Considering the productivity and efficiency of all decision making units (DMUs), that is, all TCM hospitals are actually affected by the environmental and random factors (15-18), the Bootstrap method was introduced to solve this problem (21-23). Its basic idea is to simulate the data-generating process (DGP) by repeating sample selection, thereby bias-corrected productivity and efficiency scores with Cls being generated. In result, it is much closer to the actual productivity and efficiency of TCM hospitals. Furthermore, the Bootstrap-Malmquist-DEA model can be able to infer that whether the changes of the productivity and efficiency are statistically significant.

\section{Data sources and collection}

The data of the individual tertiary public TCM hospitals were collected from official Yearbook of Traditional Chinese Medicine of China. Till now, Yearbook of Traditional Chinese Medicine of China (2018) has come out. However, the data of individual TCM hospitals were not included in this edition. Hence, the data of TCM hospitals from official Yearbook of Traditional Chinese Medicine of China from 2010 to 2017, which actually reported the data of TCM hospitals during 2009 to 2016 , were analysed in this study.

\section{Data processing}

When transferring the data collected from the Yearbook of Traditional Chinese Medicine of China (2010-2017) into the database, we found that the data of the individual tertiary public TCM hospitals in Yearbook 2013 and 2014, which reported the data of 2012 and 2013, were duplicated. By checking the relevant resources, the data in fact represented the data of 2012 and the data of 2013 were missing. Hence, the geo-means of the estimated productivity and efficiency scores between 2012 and 2014 expressed the changes between 2013 and 2014 and between 2014 and 2015. In addition, the sequence of the TCM hospitals was labelled with $\mathrm{T} 1, \mathrm{~T} 2$, and T3...

Given that it is not required that the number of individual tertiary public TCM hospitals for each year must be equal within the Bootstrap-Malmquist-DEA model, the respective actual numbers of included TCM hospitals for every two years were $69,74,65,68,81$, and 78 in this study.

\section{Statistical analysis}

SPSS 23.0 version statistical software was used to conduct the descriptive analysis of the input and output indicators. R 3.2.1 version statistical software was applied to measure the productivity and efficiency of the TCM hospitals with FEAR package (24). The number of repeating sample selection was 2000. The statistical significant was set at $P<0.05$.

\section{Results}

\section{Characteristics of the input and output indicators}

As shown in Table 1, the trends of all the input and output indicators were positively increasing from 2009 to 2016 . The annual average growth rates for each input and output indicators were 6.61\% (health professionals), $8.15 \%$ (actual open beds), $7.08 \%$ (outpatients and inpatients) and $12.50 \%$ (discharged patients) respectively from 2009 to 2016 .

Table 1 Summary of the input and output indicators of the tertiary public TCM hospitals from 2009 to 2016 


\begin{tabular}{|c|c|c|c|c|c|}
\hline \multirow[t]{3}{*}{ Year } & & \multicolumn{2}{|l|}{ Input indicators } & \multicolumn{2}{|l|}{ Output indicators } \\
\hline & & \multicolumn{4}{|c|}{ Mean \pm Standard Deviation } \\
\hline & & $\begin{array}{l}\text { Number of health } \\
\text { professionals }\end{array}$ & $\begin{array}{l}\text { Number of actual open } \\
\text { beds }\end{array}$ & $\begin{array}{l}\text { Number of outpatients and } \\
\text { inpatients }\end{array}$ & $\begin{array}{l}\text { Number of discharged } \\
\text { patients }\end{array}$ \\
\hline \multirow{2}{*}{$\begin{array}{l}2009- \\
2010\end{array}$} & 2009 & $920 \pm 509$ & $664 \pm 355$ & $762,214 \pm 758,445$ & $14,256 \pm 9,808$ \\
\hline & 2010 & $974 \pm 550$ & $729 \pm 388$ & $847,459 \pm 827,041$ & $16,826 \pm 11,447$ \\
\hline \multirow{2}{*}{$\begin{array}{l}2010- \\
2011\end{array}$} & 2010 & $944 \pm 532$ & $701 \pm 384$ & $822,804 \pm 815,199$ & $16,078 \pm 11,291$ \\
\hline & 2011 & $1010 \pm 562$ & $784 \pm 453$ & $931,495 \pm 911,056$ & $18,505 \pm 12,796$ \\
\hline \multirow{2}{*}{$\begin{array}{l}2011- \\
2012\end{array}$} & 2011 & $1016 \pm 572$ & $753 \pm 414$ & $972,100 \pm 956,064$ & $17,628 \pm 11,371$ \\
\hline & 2012 & $1099 \pm 602$ & $819 \pm 457$ & $1,086,060 \pm 1,037,988$ & $20,465 \pm 13,121$ \\
\hline \multirow{2}{*}{$\begin{array}{l}2012- \\
2014\end{array}$} & 2012 & $1085 \pm 604$ & $815 \pm 475$ & $998,471 \pm 997,479$ & $20,565 \pm 14,833$ \\
\hline & 2014 & $1249 \pm 687$ & $977 \pm 554$ & $1,173,266 \pm 1,121,291$ & $26,573 \pm 18,727$ \\
\hline \multirow{2}{*}{$\begin{array}{l}2014- \\
2015\end{array}$} & 2014 & $1235 \pm 686$ & $978 \pm 547$ & $1,120,791 \pm 1,092,796$ & $26,598 \pm 18,211$ \\
\hline & 2015 & $1324 \pm 760$ & $1065 \pm 592$ & $1,151,680 \pm 1,100,381$ & $28,453 \pm 19,500$ \\
\hline \multirow{2}{*}{$\begin{array}{l}2015- \\
2016\end{array}$} & 2015 & $1341 \pm 765$ & $1071 \pm 596$ & $1,174,471 \pm 1,113,654$ & $28,840 \pm 19,527$ \\
\hline & 2016 & $1440 \pm 765$ & $1149 \pm 634$ & $1,230,698 \pm 1,138,641$ & $32,509 \pm 21,606$ \\
\hline
\end{tabular}

Summary of the individual changes of the total factor productivity and its decomposition index of the tertiary public TCM hospitals

Appendix 1-6 describe the results of the changes of the total factor productivity and its decomposition index of the individual tertiary public TCM hospitals. Except the TFPC between 2014 and 2015, more than half of the TCM hospitals had TFPC scores over 1.000, indicating increased productivity.

Table 2 Summary of the changes of the TFP and its decomposition index of the individual tertiary public TCM hospitals from 2009 to 2016

\begin{tabular}{|c|c|c|c|c|c|c|c|}
\hline \multicolumn{2}{|c|}{ Productivity and efficiency } & 2009-2010 & 2010-2011 & 2011-2012 & 2012-2014 & 2014-2015 & 2015-2016 \\
\hline & & \multicolumn{6}{|l|}{$N(\%)$} \\
\hline \multirow[t]{3}{*}{ TFPC } & $>1.000$ & $51(75.00)$ & $51(68.92)$ & $54(83.08)$ & $52(76.47)$ & $36(44.44)$ & $53(67.95)$ \\
\hline & $=1.000$ & $0(0.00)$ & $0(0.00)$ & $0(0.00)$ & $0(0.00)$ & $0(0.00)$ & $1(1.28)$ \\
\hline & $<1.000$ & $18(25.00)$ & $23(31.08)$ & $11(16.92)$ & $16(23.53)$ & $45(55.56)$ & $24(30.77)$ \\
\hline \multirow[t]{3}{*}{ TEC } & $>1.000$ & $45(65.22)$ & $54(72.97)$ & $27(41.54)$ & $33(48.53)$ & $35(43.21)$ & $56(71.79)$ \\
\hline & $=1.000$ & $2(2.90)$ & $3(4.06)$ & $5(7.69)$ & $3(4.41)$ & $3(3.70)$ & $4(5.13)$ \\
\hline & $<1.000$ & $22(31.88)$ & $17(22.97)$ & $33(50.77)$ & $32(47.06)$ & $43(53.09)$ & $18(23.08)$ \\
\hline \multirow[t]{3}{*}{ TC } & $>1.000$ & $45(66.18)$ & $33(44.59)$ & $65(100.00)$ & $63(92.65)$ & $51(62.96)$ & $34(43.59)$ \\
\hline & $=1.000$ & $0(0.00)$ & $0(0.00)$ & $0(0.00)$ & $0(0.00)$ & $0(0.00)$ & $0(0.00)$ \\
\hline & $<1.000$ & $24(38.82)$ & $41(55.41)$ & $0(0.00)$ & $5(7.35)$ & $30(37.04)$ & $44(56.41)$ \\
\hline \multirow[t]{3}{*}{ PTEC } & $>1.000$ & $41(59.42)$ & $43(58.11)$ & $22(33.85)$ & $37(54.41)$ & $35(43.21)$ & $49(62.82)$ \\
\hline & $=1.000$ & $6(8.70)$ & $9(12.16)$ & $11(16.92)$ & $6(8.82)$ & $6(7.41)$ & $9(11.11)$ \\
\hline & $<1.000$ & $22(31.88)$ & $22(29.73)$ & $32(49.23)$ & $25(36.77)$ & $40(49.38)$ & $20(26.07)$ \\
\hline \multirow[t]{3}{*}{ SEC } & $>1.000$ & 31 (44.93) & $55(74.32)$ & 18 (27.69) & $14(20.59)$ & $25(30.87)$ & $42(53.85)$ \\
\hline & $=1.000$ & $2(2.90)$ & $3(4.06)$ & 5 (7.69) & $3(4.41)$ & $3(3.70)$ & $4(5.13)$ \\
\hline & $<1.000$ & $36(52.17)$ & $16(21.62)$ & $42(64.62)$ & $51(75.00)$ & $53(65.43)$ & $32(41.02)$ \\
\hline
\end{tabular}

Summary of the annual geo-means of the changes of the total factor productivity and its decomposition index of the tertiary public TCM hospitals

Table 3 shows that the overall annual geo-means of the scores of the TFPC and its decomposition index were 1.0379 (TFPC), 1.0167 (TEC), 1.0209 (TC), 1.0212 (PTEC), and 0.9955 (SEC) respectively (Table 2). Combined with Figure 1, the trend of the TFPC of the TCM hospitals was slightly fluctuant. Also, except the TFPC between 2014 and 2015, the rest of the TFPC were slightly increasing. Additionally, from 2011 to 2014 , the main factor as the driver to improve the TFP was the technology positive progress. In contrast, both the technology positive progress and the increased technological efficiency improved 
the TFP between 2009 and 2010 while the technological positive efficiency was mainly the factor to improve the TFP between 2015 and 2016 . From 2011 to 2015, the TEC were decreasing due to the decreased SEC.

Table 3 Summary of the annual geo-means of the changes of the TFP and its decomposition index of the tertiary public TCM hospitals from 2009 to 2016

\begin{tabular}{|llllll|}
\hline Year & TFPC & TEC & TC & PTEC & SEC \\
\hline $\mathbf{2 0 0 9 - 2 0 1 0}$ & 1.0621 & 1.0356 & 1.0256 & 1.0434 & 0.9926 \\
\hline $\mathbf{2 0 1 0 - 2 0 1 1}$ & 1.0484 & 1.0553 & 0.9935 & 1.0274 & 1.0272 \\
\hline $\mathbf{2 0 1 1 - 2 0 1 2}$ & 1.0692 & 0.9836 & 1.0869 & 1.0053 & 0.9785 \\
\hline $\mathbf{2 0 1 2 - 2 0 1 3}$ & $1.0459^{*}$ & $0.9985^{\star}$ & $1.0474^{\star}$ & $1.0130^{*}$ & $0.9857^{\star}$ \\
\hline $\mathbf{2 0 1 3 - 2 0 1 4}$ & $1.0459^{*}$ & $0.9985^{\star}$ & $1.0474^{\star}$ & $1.0130^{*}$ & $0.9857^{\star}$ \\
\hline $\mathbf{2 0 1 4 - 2 0 1 5}$ & 0.9756 & 0.9731 & 1.0025 & 0.9821 & 0.9908 \\
\hline $\mathbf{2 0 1 5 - 2 0 1 6}$ & 1.0214 & 1.0763 & 0.9490 & 1.0667 & 1.0090 \\
\hline Geo-mean & 1.0379 & 1.0167 & 1.0209 & 1.0212 & 0.9955 \\
\hline
\end{tabular}

Note*: It means that the geo-means of the scores of the TFP and its decomposition index between 2012 and 2014.

\section{Discussion}

Our study showed that the overall annual rate of the total factor productivity of the tertiary public TCM hospitals was slightly increased because of the increasing invest by the Chinese government. The technological progress was the main driver to improve the total factor productivity, which was associated with the innovation of the TCM technology. The decreased TE was more affected by the decreased SE, indicating the restriction of the scale of the TCM hospitals.

Our study found that the whole annual increasing rate of the TFP was 3.79\%, which was a slight growth. This is mainly because after the new medical reform was published, the Chinese government has extensively invested on the development of the public hospitals, including the TCM hospitals. This result was different from that of Yang's study (4). It estimated that the overall rate of the TFP of the TCM hospitals was decreased from 2012 to 2016 , which was $1.30 \%$. It may be because of the different DMUs. Though both of the two studies measured the productivity and efficiency of the TCM hospitals at a national level, our study adopted the individual tertiary public TCM hospitals as the DMUs while all levels of TCM hospitals in a province were the DMUs in Yang's study. Compared with the secondary and primary TCM hospitals, the tertiary public TCM hospitals are equipped with more advanced medical instruments, more excellent health professionals and better management systems, thereby leading to Siphon effect on patients (25). This could result in higher productivity and efficiency for the tertiary public TCM hospitals. It may also be because the different input and output indicators were selected in the two studies and Yang's study employed the classical Malmquist-DEA model without introducing the Bootstrap method. These factors could result in different results.

Although the overall TFP rates had increased except the 2014-2015 period, technological progress was found to be the main driver to improve the TFP. The high medical technology is one of the prominent components of the high quality of the medical services and can attract more and more patients to seek medical services. Generally, the TCM hospitals cannot be comparable to the general hospitals, which concentrate on the western medical services $(4,5)$. Therefore, the TCM hospitals especially need to pay attention to the development and innovation of the TCM technology, thereby improving the competitiveness. Also, the development of the TCM technology includes strengthening the training of the current TCM human resources (8). Consequently, the whole level of the TCM technology of a TCM hospital can be improved and then improve the productivity and efficiency of the TCM hospitals.

The TFPC between 2014 and 2015 was 0.9756 , which was influenced more by the decreased TE. Furthermore, the decreased TE was more affected by the decreased SE. This indicated that the scale of the tertiary public TCM hospitals should be restricted in order to be blindly extended. The hospital managers should pursuit the high quality, high efficiency and low cost of the TCM medical services rather than the high volume. This can be achieved by transferring the traditional and single management system to a more refined management system, which includes strengthening the hospital financial system, improving the hospital environment and adjusting the price of the TCM services (26).

High productivity and efficiency of the TCM hospitals is highly related to the rational use of health resources (14). The rational use of health resources can be up to the well-structured health professionals (17). Even though this study showed that the number of health professionals was increasing yearly, it could not infer whether the structure of the health professionals was appropriate, especially the ratio between the western clinical doctors/pharmacists and the TCM doctors/pharmacists. Hong analysed the trend of the TCM human resources at a national level from 2010 to 2015 and suggested that the number of the TCM human resources was also continuously increasing (6). However, the increased speed of the TCM pharmacists was slow and the number of the TCM human resources in the TCM hospitals was not enough. What is worse was that the loss of the TCM human resources was also a serious problem. These could have a negative effect on the development of the TCM hospitals and then deteriorate the productivity and efficiency of the TCM hospitals. Although this study showed a slightly increased TFP of the TCM hospitals, it cannot be denied that the TCM hospitals have to strengthen the management level of the TCM human resources. Fang proposed that in order to avoid the loss of the hospital human resources, it was necessary to rebuild the performance and salary management system based on the Diagnosis Related Groups (DRGs) system (25). Specifically, the DRGs could identify the difficulty and scope of the medical services and help assess the medical services. Then, a more advanced performance and salary system can be able to improve the motivation of the TCM human resources to keep them from quitting a job. 
In order to keep improving the productivity and efficiency of the tertiary public TCM hospitals, it is imperative for the TCM hospitals to actively participate in the hierarchical diagnosis and treatment system to better paly am important role on the medical alliance $(25,27)$. This can realize the rational use of TCM resources in some way, thereby improving the productivity and efficiency of the tertiary public TCM hospitals based on the abovementioned analysis. However, Wang's study showed that the hierarchical diagnosis and treatment system has not been established within the TCM medical alliance, because it is always the first choice for the patients to go to the tertiary TCM hospitals to seek the medical services and the ability of a secondary or primary TCM hospital is not enough to meet the patients' medical demands (27). Additionally, to better establish the hierarchical diagnosis and treatment system is associated with the well-established hospital information system (28). It can be able to realize the TCM resource sharing to further achieve the rational use of the TCM resources.

\section{Limitations}

This study made a contribution to the current studies by applying a more accurate method (the Bootstrap-Malmquist-DEA model) to measure the productivity and efficiency of the individual tertiary public TCM hospitals at a national level. However, there are still some limitations. First, this study did not include the input and output indicators which represent the characteristics of the TCM medical services. There was no information reported by the official Yearbook of Traditional Chinese Medicine of China. It will be better to ask for the information of the relevant indicators from the individual TCM hospitals to further directly analyse the factors which have an impact on the productivity and efficiency of the TCM hospitals. Second, this study only analysed the productivity and efficiency of the TCM hospitals. However, it did not explain the relationship between the quality and the productivity and efficiency, as well as the relationship between the cost and the productivity and efficiency. Quality and cost should be essential part of the further research. Third, due to the duplicated data from 2012 to 2013, it was not clear about the actual productivity and efficiency between 2012 and 2013 and between 2013 and 2014 . It should improve the quality of the data collection in the future.

\section{Conclusions}

The overall annual rate of the total factor productivity of the tertiary public TCM hospitals was slightly increased. The technological progress was the main driver to improve the total factor productivity. The TCM hospitals need to pay attention to the development and innovation of the TCM technology, thereby improving the competitiveness. The hospital managers should pursuit the high quality, high efficiency and low cost of the TCM medical services. It is necessary to rebuild the performance and salary management system based on the DRGs system. It is imperative for the TCM hospitals to actively participate in the hierarchical diagnosis and treatment system to better paly am important role on the medical alliance and to better establish the hospital information system to realize the TCM resource sharing.

\section{Abbreviations}

Cls: Confidence Intervals

DEA: Data Envelopment Analysis

DGP: Data-Generating Process

DMUs: Decision-Making Units

DRGs: Diagnosis Related Groups

MPI: Malmquist Productivity Index

PTEC: Pure Technical efficiency Changes

SEC: Scale Efficiency Changes

TC: Technological Changes

TCM: Traditional Chinese Medicine

TEC: Technical Efficiency Changes

TFPC: Total Factor Productivity Changes

\section{Declarations}

\section{Ethics approval and consent to participate}

Data were all from the official report and did not include the personal information of humans. Therefore, there were no needs to ask for ethics approval and consent to participate.

\section{Consent for publication}

All the authors listed in this paper have already read this manuscript and agreed to submit the paper and for later publication.

\section{Availability of data and materials}


Data and materials will be accessible with the permission from the research team.

\section{Competing interests}

The authors declare that they have no competing interests.

\section{Funding}

It is not applicable.

\section{Code availability}

We have got the approval of FEAR package use from Professor Wilson.

\section{Authors' contributions}

X.L. processed and analysed the data and wrote the draft of the manuscript. R.C. helped collect the data. S.P. did the data entry. Q.W. and J.W. gave the comments on the manuscript for further improvement. J.L. helped the submission of the manuscript. H.L. designed the study and made the comments on the manuscript for better quality.

\section{Acknowledgements}

We would like to thank Rui Chen, the staff from the National Administration of Traditional Chinese Medicine for helping collect the data.

\section{References}

1. China's Central Party Committee and the State Council. Healthy China 2030 (from vision to action) . 2016 [cited January 10, 2020]. Available from: https://www.who.int/healthpromotion/conferences/9gchp/healthy-china/en/.

2. Shen P, Geng R, Shou W, Ban L, Li R. Study on the regional allocation analysis of health resources of Traditional Chinese Medicine Hospitals (in Chinese). Chinese Journal of Health Statistics. 2018;35(3):445-8.

3. Wang X, Zhang A, Sun H, Yan G, Wang P, Han Y. Traditional chinese medicine: current state, challenges, and applications. Serum Pharmacochemistry of Traditional Chinese Medicine: Elsevier; 2017. p. 1-6.

4. Yang X, Zhu C. Study on regional differences and cooperative development of service efficiency of Chinese medicine hospitals in China (in Chinese). Health Economics Research. 2019;36(12):25-8.

5. Wang L, Suo S, Li J, Hu Y, Li P, Wang Y, et al. An investigation into traditional chinese medicine hospitals in China: Development trend and medical service innovation. International Journal of Health Policy Management. 2017;6(1):19.

6. Xu Y, Hong B, Li Z, Hu Y. Analysis and development prediciton of current situation of TCM human resources (in Chinese). Chinese Journal of Information on Traditional Chinese Medicine. 2018;25(6):1-5.

7. Hong B, Fang Y, Cheng W, Man X, Shi X. The status quo and problems of traditional Chinese medicine service system in China (In Chinese). Chinese Health Economics. 2010;29(9):33-5.

8. Ding F, Cheng W, Fang Y, Cheng Z, Shi S, Shi X. Study on the Malmquist Index of operating efficiency in tertiary traditional Chinese medicine hospitals in Beijing (in Chinese). Chinese Hospital Management. 2016;36(7):55-7.

9. Dong S, Zuo Y, Guo S, Li M, Liu X, Li H. Data envelopment analysis for relative efficiency measurements of Chinese hospitals: a systematic review. Research in Health Science. 2017;2(2):79-103.

10. Färe R, Grosskopf S, Norris M, Zhang Z. Productivity growth, technical progress, and efficiency change in industrialized countries. The American economic review. 1994:66-83.

11. Masri MD, Asbu EZ. Productivity change of national health systems in the WHO Eastern Mediterranean region: application of DEA-based Malmquist productivity index. Global Health Research and Policy. 2018;3(1):22.

12. Wang Z, Ding F, Ma C, Yuan S, Shi X, Shi S. Study on the Malmquist Index of secondary TCM hospitals TFP in Beijing (in Chinese). Chinese Hospital Management. 2016;36(10):50-2.

13. Kang L, Kraipornsak P. Technical and Scale Efficiency of Traditional Medicine Hospitals and General Hospitals in Inner Mongolia, China. Souteast Asian Journal of Economics. 2016:105-37.

14. Zhang P, Deng W, Mao S, Chang K, editors. Evaluation of Health Resource Allocation Efficiency of Chinese Medicine Hospital Based on Data Envelopment Analysis. 2nd International Conference on Social Science, Public Health and Education (SSPHE 2018); 2019: Atlantis Press.

15. Simar L, Wilson PW. Sensitivity analysis of efficiency scores: How to bootstrap in nonparametric frontier models. Management Science. 1998;44(1):49-61.

16. Simar L, Wilson PW. A general methodology for bootstrapping in non-parametric frontier models. Journal of Applied Statistics. 2000;27(6):779-802.

17. Liu X, Wang Q, Sara B, Yang W, Dong S, Li H. The Technical Efficiency of Community Health Service Centers in Wuhan, China: Estimation and Policy Implications. INQUIRY: The Journal of Health Care Organization, Provision,Financing. 2018;55:0046958018812972.

18. Li H, Dong S. Measuring and Benchmarking Technical Efficiency of Public Hospitals in Tianjin, China: A Bootstrap-Data Envelopment Analysis Approach. INQUIRY: The Journal of Health Care Organization, Provision, and Financing. 2015;52:0046958015605487. 
19. Chen Y, Liu X, Deng G, Wang Q, Li H. Measuring technical efficiency of township hospitals in Wuhan: A Bootstrap-DEA approach (in Chinese). Chinese Rural Health Service Administration. 2017;37(6):648-52.

20. Caves DW, Christensen LR, Diewert WE. The economic theory of index numbers and the measurement of input, output, and productivity. Econometrica: Journal of the Econometric Society. 1982:1393-414.

21. Lothgren M, Tambour M. Bootstrapping the data envelopment analysis Malmquist productivity index. Applied Economics. 1999;31(4):417-25.

22. Daraio C, Simar L. Advanced robust and nonparametric methods in efficiency analysis: Methodology and applications: Springer Science \& Business Media; 2007.

23. Efron B. Bootstrap methods: another look at the jackknife. Breakthroughs in statistics: Springer; 1992. p. 569-93.

24. Wilson PW. FEAR: A software package for frontier efficiency analysis with R. Socio-economic Planning Sciences. 2008;42(4):247-54.

25. Fang P, Chen J, Liu S, Zhang Q. Challenges and reflection on challenges of public hospitals under the hierachical diagnosis and treatment system (in Chinese). Chinese Hospital Management. 2018;38(10):1-3.

26. Xi H, Kong F, Xie J, Ma A, Tang W. Influence of pharmaceutical pricing reform on economic operating efficiency of urban public hospitals (in Chinese). Chinese Hospital Management. 2018;38(4):15-9.

27. Wang B. Challenge and consideration of traditional Chinese medicine development in the construction of medical alliance (in Chinese). Soft Science of Health. 2019;33(12):18-20.

28. Sun W, Sun Q. Thoughts on the practice method of public hospitals under herachical diagnosis (in Chinese). Chinese Primary Health Care. 2019;33(5):1-3.

29. The important role of polysaccharides from a traditional Chinese medicine-Lung Cleansing and Detoxifying Decoction against the COVID-19 pandemic.

\section{Appendices}

Appendix 1 Results of the changes of the TFP and its decomposition index of the individual tertiary public TCMHs from 2009 to 2010 


\begin{tabular}{|c|c|c|c|c|c|c|c|c|c|c|c|c|c|c|}
\hline \multirow{2}{*}{$\begin{array}{l}\text { TCMH } \\
\text { NO }\end{array}$} & \multirow[t]{2}{*}{ TFPC } & \multirow[b]{2}{*}{$\begin{array}{l}\text { Lower } \\
\text { Bounds }\end{array}$} & \multicolumn{3}{|c|}{ TEC } & \multicolumn{3}{|c|}{ TC } & \multicolumn{3}{|c|}{ PTEPC } & \multicolumn{3}{|c|}{ SEC } \\
\hline & & & $\begin{array}{l}\text { Upper } \\
\text { Bounds }\end{array}$ & & $\begin{array}{l}\text { Lower } \\
\text { Bounds }\end{array}$ & $\begin{array}{l}\text { Upper } \\
\text { Bounds }\end{array}$ & & $\begin{array}{l}\text { Lower } \\
\text { Bounds }\end{array}$ & $\begin{array}{l}\text { Upper } \\
\text { Bounds }\end{array}$ & & $\begin{array}{l}\text { Lower } \\
\text { Bounds }\end{array}$ & $\begin{array}{l}\text { Upper } \\
\text { Bounds }\end{array}$ & & $\mathrm{L}$ \\
\hline T1 & 1.0037 & 0.9398 & 1.0057 & 1.0217 & 0.9281 & 1.1478 & 0.9825 & 0.8212 & 1.0448 & 0.9776 & 0.8554 & 1.1198 & 1.0451 & 0 \\
\hline T2 & $1.0900^{*}$ & 1.0814 & 1.0900 & $1.1064^{*}$ & 1.0198 & 1.2891 & 0.9852 & 0.7856 & 1.0536 & 0.8919 & 0.6489 & 1.0477 & $1.2406^{*}$ & 1 \\
\hline T3 & $1.1219 *$ & 1.1118 & 1.1241 & 1.0611 & 0.8845 & 1.2055 & 1.0573 & 0.8834 & 1.2040 & 1.0494 & 0.8558 & 1.1832 & 1.0111 & 0 \\
\hline T4 & 1.1899* & 1.1795 & 1.2122 & $1.1692^{*}$ & 1.0086 & 1.3682 & 1.0177 & 0.8109 & 1.1395 & 1.1456 & 0.9544 & 1.3205 & 1.0206 & 0 \\
\hline T5 & $1.1113^{*}$ & 1.0849 & 1.1229 & $1.1586 *$ & 1.1027 & 1.2887 & $0.9591 *$ & 0.8284 & 0.9943 & 1.0805 & 0.9916 & 1.1891 & 1.0723 & 0 \\
\hline T6 & 1.0399* & 1.0379 & 1.0737 & 0.9250 & 0.7370 & 1.0146 & $1.1243^{*}$ & 1.0141 & 1.3162 & 0.9556 & 0.8003 & 1.1122 & 0.9679 & 0 \\
\hline T7 & $1.0841 *$ & 1.0620 & 1.1649 & $1.0811^{*}$ & 1.0185 & 1.2481 & 1.0028 & 0.8714 & 1.0662 & $1.0987^{*}$ & 1.0454 & 1.2768 & 0.9840 & 0 \\
\hline T8 & $1.2840 *$ & 1.2783 & 1.3831 & $1.2374^{*}$ & 1.0597 & 1.5283 & 1.0377 & 0.7729 & 1.1839 & $1.2758 *$ & 1.1203 & 1.6080 & $0.9698 *$ & 0 \\
\hline T9 & 0.9937 & 0.9609 & 1.0198 & 0.9427 & 0.8288 & 1.0554 & 1.0541 & 0.9132 & 1.1607 & 0.9529 & 0.8428 & 1.0794 & 0.9893 & 0 \\
\hline T10 & 0.9794 & 0.9667 & 1.0322 & 0.9113 & 0.7663 & 1.0535 & 1.0747 & 0.9088 & 1.2419 & 1.0000 & 0.5851 & 1.3278 & 0.9113 & 0 \\
\hline T11 & $1.0363^{*}$ & 1.0303 & 1.0504 & 1.0103 & 0.9216 & 1.1133 & 1.0257 & 0.9120 & 1.1115 & 1.0614 & 0.9141 & 1.1981 & 0.9519 & 0 \\
\hline T12 & 1.2240 * & 1.2088 & 1.2469 & $1.2209 *$ & 1.1431 & 1.3491 & 1.0026 & 0.8894 & 1.0666 & 1.0000 & -0.2599 & 1.2192 & 1.2209 & 0 \\
\hline T13 & $1.0526^{*}$ & 1.0524 & 1.0530 & $1.0742^{*}$ & 1.0207 & 1.1861 & 0.9799 & 0.8658 & 1.0264 & 1.0073 & 0.8281 & 1.1569 & 1.0664 & 0 \\
\hline T14 & $1.0963^{*}$ & 1.0734 & 1.1170 & $1.1144^{*}$ & 1.0179 & 1.2476 & 0.9837 & 0.8497 & 1.0628 & 1.0366 & 0.8883 & 1.2172 & 1.0750 & 0 \\
\hline T15 & $1.0963^{*}$ & 1.0914 & 1.1477 & 1.0354 & 0.9240 & 1.1763 & 1.0588 & 0.9229 & 1.1804 & 1.0621 & 0.8647 & 1.2813 & 0.9749 & 0 \\
\hline T16 & $1.4056^{*}$ & 1.3874 & 1.4334 & $1.3776^{*}$ & 1.1932 & 1.5207 & 1.0203 & 0.9004 & 1.1367 & $1.4976^{*}$ & 1.3876 & 1.7067 & 0.9199* & 0 \\
\hline T17 & 1.1830 * & 1.1817 & 1.2717 & $1.1924^{*}$ & 1.1232 & 1.4103 & 0.9922 & 0.8201 & 1.0631 & $1.1861^{*}$ & 1.0965 & 1.3874 & 1.0053 & 0 \\
\hline T18 & $1.1157^{*}$ & 1.1091 & 1.1213 & $1.1438 *$ & 1.0452 & 1.2799 & 0.9755 & 0.8426 & 1.0521 & 1.3250 * & 1.1950 & 1.4917 & $0.8632^{*}$ & 0 \\
\hline T19 & 0.9835 & 0.9787 & 1.0105 & 1.0080 & 0.9525 & 1.1708 & 0.9757 & 0.7976 & 1.0315 & 1.0245 & 0.9557 & 1.1792 & 0.9839 & 0 \\
\hline T20 & 1.0114 & 0.9855 & 1.0117 & 0.8565 & 0.5464 & 1.1056 & 1.1808 & 0.6947 & 1.4817 & 0.8251 & 0.4973 & 1.0555 & 1.0380 & 0 \\
\hline T21 & $1.1267 *$ & 1.1253 & 1.1305 & 1.1020 & 0.9949 & 1.2291 & 1.0224 & 0.8902 & 1.1137 & $1.1393^{*}$ & 1.0308 & 1.2800 & 0.9673 & 0 \\
\hline T22 & $1.0826 *$ & 1.0548 & 1.1102 & $1.1065^{*}$ & 1.0445 & 1.2482 & 0.9784 & 0.8329 & 1.0322 & 1.0224 & 0.8172 & 1.2422 & 1.0822 & 0 \\
\hline T23 & $1.4559 *$ & 1.3901 & 1.5282 & $1.4364^{*}$ & 1.2862 & 1.6905 & 1.0136 & 0.8009 & 1.1049 & $1.4115^{\star}$ & 1.2083 & 1.7090 & 1.0176 & 0 \\
\hline T24 & $1.0513^{*}$ & 1.0135 & 1.0648 & 1.0069 & 0.8080 & 1.2301 & 1.0441 & 0.7362 & 1.2039 & 1.0306 & 0.8922 & 1.2872 & 0.9771 & 0 \\
\hline T25 & $1.0765^{\star}$ & 1.0622 & 1.0788 & $1.0895^{\star}$ & 1.0016 & 1.2313 & 0.9881 & 0.8359 & 1.0586 & 1.0777 & 0.9866 & 1.2180 & 1.0109 & 0 \\
\hline T26 & $1.0669 *$ & 1.0626 & 1.0791 & $1.1200 *$ & 1.0698 & 1.2691 & $0.9526 *$ & 0.8089 & 0.9973 & $1.0811^{*}$ & 1.0000 & 1.1746 & 1.0359 & 0 \\
\hline T27 & 2.1700 * & 2.0142 & 2.3349 & $2.0449 *$ & 1.7864 & 2.3870 & 1.0612 & 0.8579 & 1.1797 & $1.7953^{*}$ & 1.4246 & 2.0120 & 1.1390 * & 1 \\
\hline T28 & $0.7021^{*}$ & 0.5423 & 0.7021 & $0.6679 *$ & 0.4744 & 0.7373 & 1.0512 & 0.8483 & 1.1964 & $0.6714^{*}$ & 0.4219 & 0.7621 & 0.9948 & 0 \\
\hline T29 & 0.9943 & 0.9849 & 1.0129 & 1.0006 & 0.9122 & 1.1239 & 0.9937 & 0.8582 & 1.0769 & $1.1874^{*}$ & 1.1051 & 1.4052 & $0.8426^{*}$ & 0 \\
\hline Т30 & 0.9969 & 0.9625 & 1.0445 & 0.9129 & 0.6745 & 1.1728 & 1.0919 & 0.6965 & 1.3115 & 0.9280 & 0.7167 & 1.1770 & 0.9837 & 0 \\
\hline Т31 & $1.1224^{*}$ & 1.1216 & 1.1276 & $1.1735^{\star}$ & 1.1212 & 1.3317 & $0.9565^{\star}$ & 0.8078 & 0.9988 & $1.1046 *$ & 1.0176 & 1.2201 & 1.0623 & 0 \\
\hline T32 & $0.9379 *$ & 0.7819 & 0.9598 & 0.9494 & 0.7996 & 1.0506 & 0.9880 & 0.8188 & 1.0513 & 1.0868 & 0.9774 & 1.2805 & $0.8735^{\star}$ & 0 \\
\hline T33 & $0.4894^{*}$ & 0.4731 & 0.5993 & $0.5496 *$ & 0.5047 & 0.7538 & $0.8904^{*}$ & 0.6280 & 0.9592 & 0.7258 & 0.6049 & 1.1994 & $0.7573^{*}$ & -( \\
\hline T34 & $1.0804^{*}$ & 1.0730 & 1.0924 & $1.1643^{*}$ & 1.1385 & 1.3300 & $0.9280 *$ & 0.7720 & 0.9495 & $1.1498^{*}$ & 1.0460 & 1.2362 & $1.0126^{*}$ & 1 \\
\hline T35 & $1.0427 *$ & 1.0156 & 1.0989 & 0.9965 & 0.8510 & 1.1522 & 1.0464 & 0.8650 & 1.1830 & 1.0000 & 0.7391 & 1.2061 & 0.9965 & 0 \\
\hline T36 & $0.9759 *$ & 0.9733 & 0.9790 & 1.0105 & 0.9456 & 1.1369 & 0.9657 & 0.8292 & 1.0243 & 0.9902 & 0.9149 & 1.0876 & 1.0205 & 0 \\
\hline T37 & $0.8015^{\star}$ & 0.6640 & 0.8024 & $0.7691^{*}$ & 0.5870 & 0.8345 & 1.0421 & 0.8861 & 1.1743 & $0.8639 *$ & 0.7360 & 0.9920 & $0.8903^{*}$ & 0 \\
\hline T38 & $1.1394^{*}$ & 1.1394 & 1.1804 & 1.0839 & 0.9090 & 1.3055 & 1.0512 & 0.8033 & 1.2040 & 1.0000 & 0.4195 & 1.4543 & 1.0839 & 0 \\
\hline Т39 & $0.8527 *$ & 0.8370 & 0.8735 & 0.9129 & 0.8868 & 1.0528 & $0.9340 *$ & 0.7845 & 0.9609 & $0.7982^{*}$ & 0.6770 & 0.8660 & $1.1436 *$ & 1 \\
\hline T40 & $0.8476^{*}$ & 0.7697 & 0.8517 & $0.8323^{*}$ & 0.6992 & 0.9141 & 1.0184 & 0.8734 & 1.1227 & $0.8871 *$ & 0.7939 & 0.9971 & $0.9382^{*}$ & 0 \\
\hline T41 & $0.8316^{*}$ & 0.7106 & 0.8336 & $0.7962^{*}$ & 0.6192 & 0.8696 & 1.0444 & 0.8915 & 1.1784 & 0.8338 & 0.7233 & 1.0243 & 0.9550 & 0 \\
\hline
\end{tabular}




\begin{tabular}{|c|c|c|c|c|c|c|c|c|c|c|c|c|c|c|}
\hline T42 & $0.8102 *$ & 0.7501 & 0.8152 & 0.8360 * & 0.7657 & 0.9364 & 0.9691 & 0.8199 & 1.0172 & $0.8523^{*}$ & 0.7852 & 0.9507 & 0.9809 & 0 \\
\hline T43 & $1.0712^{*}$ & 1.0248 & 1.0767 & 0.9461 & 0.7629 & 1.0385 & 1.1322 & 0.9887 & 1.2999 & 0.9790 & 0.7989 & 1.1137 & 0.9664 & 0 \\
\hline T44 & $1.0592^{*}$ & 1.0267 & 1.0736 & 0.9442 & 0.7872 & 1.0377 & 1.1218 & 0.9921 & 1.2788 & 0.9517 & 0.7796 & 1.0752 & 0.9921 & 0 \\
\hline T45 & $1.3314^{*}$ & 1.2871 & 1.3875 & $1.3360^{*}$ & 1.2533 & 1.4919 & 0.9965 & 0.8854 & 1.0512 & $1.3991^{*}$ & 1.3097 & 1.5773 & 0.9549 & 0 \\
\hline T46 & $1.0739 *$ & 1.0291 & 1.1305 & 1.0000 & 0.7406 & 1.2240 & 1.0739 & 0.7807 & 1.2994 & 1.0000 & 0.6898 & 1.2218 & 1.0000 & 0 \\
\hline T47 & $1.0815^{\star}$ & 1.0566 & 1.0855 & 1.0394 & 0.9008 & 1.1598 & 1.0405 & 0.8923 & 1.1528 & 1.0100 & 0.8287 & 1.1488 & 1.0291 & 0 \\
\hline T48 & $1.2292^{*}$ & 1.2208 & 1.2558 & $1.2066^{*}$ & 1.1127 & 1.3361 & 1.0187 & 0.9069 & 1.0983 & $1.1654^{\star}$ & 1.0044 & 1.3870 & 1.0354 & 0 \\
\hline T49 & $1.1425^{\star}$ & 1.1300 & 1.1677 & 1.0119 & 0.7922 & 1.1044 & $1.1291^{*}$ & 1.0269 & 1.3360 & 1.0541 & 0.8699 & 1.1922 & 0.9600 & 0 \\
\hline T50 & $0.9232^{*}$ & 0.8324 & 0.9293 & $0.8078 *$ & 0.6020 & 0.9138 & 1.1428 & 0.9120 & 1.3326 & $0.7339 *$ & 0.5648 & 0.8234 & 1.1008 & 0 \\
\hline T51 & $1.0904^{*}$ & 1.0912 & 1.1916 & 1.0495 & 0.9841 & 1.2616 & 1.0390 & 0.8419 & 1.1409 & $0.8222^{*}$ & 0.6609 & 0.9152 & $1.2763^{*}$ & 1 \\
\hline T52 & $1.1476 *$ & 1.1425 & 1.1534 & $1.1432^{*}$ & 1.0079 & 1.2617 & 1.0039 & 0.8892 & 1.1121 & $1.2278 *$ & 1.1016 & 1.3568 & $0.9311^{*}$ & 0 \\
\hline T53 & $1.2774^{*}$ & 1.2540 & 1.3150 & 1.2164 & 0.9194 & 1.5064 & 1.0501 & 0.7483 & 1.2617 & 1.1770 & 0.8620 & 1.4301 & 1.0335 & 0 \\
\hline T54 & $1.2098 *$ & 1.1750 & 1.2140 & 1.1425 & 0.9216 & 1.3098 & 1.0588 & 0.8649 & 1.2170 & 1.1747 & 0.9379 & 1.3713 & 0.9726 & 0 \\
\hline T55 & $1.1434^{*}$ & 1.1354 & 1.1633 & 1.0249 & 0.8434 & 1.1154 & $1.1156^{*}$ & 1.0151 & 1.2919 & 1.0990 & 0.8811 & 1.3347 & 0.9326 & 0 \\
\hline T56 & $1.1005^{*}$ & 1.0888 & 1.1033 & $1.1255^{\star}$ & 1.0359 & 1.2537 & 0.9778 & 0.8505 & 1.0429 & $1.4149 *$ & 1.1598 & 1.6701 & $0.7955^{\star}$ & 0 \\
\hline T57 & $1.0227^{*}$ & 1.0210 & 1.0244 & 1.0255 & 0.9123 & 1.1369 & 0.9972 & 0.8758 & 1.0960 & $1.3517 *$ & 1.1503 & 1.5722 & $0.7586 *$ & 0 \\
\hline T58 & $1.1370 *$ & 1.0404 & 1.1432 & 1.0000 & 0.6166 & 1.2755 & 1.1370 & 0.6932 & 1.4027 & 1.0000 & 0.6257 & 1.2907 & 1.0000 & 0 \\
\hline T59 & $1.1010 *$ & 1.0948 & 1.1198 & $1.1242 *$ & 1.0548 & 1.2982 & 0.9794 & 0.8047 & 1.0376 & $1.1437^{*}$ & 1.0586 & 1.3039 & 0.9830 & 0 \\
\hline T60 & $0.9333^{*}$ & 0.7796 & 0.9456 & 0.9294 & 0.7742 & 1.0238 & 1.0042 & 0.8484 & 1.0608 & $0.8669 *$ & 0.6344 & 0.9174 & $1.0721^{*}$ & 1 \\
\hline T61 & $0.9895^{*}$ & 0.9585 & 0.9986 & $0.8753^{*}$ & 0.7065 & 0.9701 & 1.1305 & 0.9913 & 1.3024 & 0.9174 & 0.7539 & 1.0439 & 0.9541 & 0 \\
\hline T62 & $0.6081 *$ & 0.5045 & 0.6368 & $0.5868 *$ & 0.4598 & 0.6536 & 1.0364 & 0.9030 & 1.1150 & $0.5520 *$ & 0.3958 & 0.5914 & $1.0629 *$ & 1 \\
\hline T63 & $1.0392^{\star}$ & 1.0370 & 1.0536 & 0.9860 & 0.8737 & 1.1144 & 1.0540 & 0.9024 & 1.1641 & 1.0080 & 0.8320 & 1.2181 & 0.9781 & 0 \\
\hline T64 & $1.1253^{*}$ & 1.0987 & 1.1273 & 1.0626 & 0.7706 & 1.2882 & 1.0590 & 0.7617 & 1.2725 & 1.0135 & 0.6921 & 1.2043 & 1.0484 & 0 \\
\hline T65 & 1.0146 & 0.9811 & 1.0229 & 0.9840 & 0.8623 & 1.0937 & 1.0311 & 0.8882 & 1.1303 & 0.9650 & 0.8135 & 1.0587 & 1.0197 & 0 \\
\hline T66 & 1.0644 & 0.9982 & 1.0979 & 1.0549 & 0.8633 & 1.2380 & 1.0090 & 0.7644 & 1.1380 & 1.0517 & 0.8337 & 1.2315 & 1.0030 & 0 \\
\hline T67 & $1.0884^{*}$ & 1.0712 & 1.1304 & $1.1114^{*}$ & 1.0665 & 1.2565 & 0.9793 & 0.8516 & 1.0221 & 1.1093 & 0.6411 & 1.3631 & 1.0019 & 0 \\
\hline T68 & $1.1178 *$ & 1.0981 & 1.1256 & $1.1045^{\star}$ & 1.0107 & 1.2083 & 1.0121 & 0.9011 & 1.0865 & $1.2346^{*}$ & 1.0601 & 1.4132 & 0.8946 & 0 \\
\hline T69 & $1.8870 *$ & 1.7519 & 2.0449 & $1.8543^{*}$ & 1.6754 & 2.1057 & 1.0176 & 0.8987 & 1.0889 & $1.9658 *$ & 1.8605 & 2.2843 & $0.9433^{*}$ & 0 \\
\hline $\begin{array}{l}\text { Geo- } \\
\text { mean }\end{array}$ & 1.0621 & - & - & 1.0356 & - & - & 1.0256 & - & - & 1.0434 & - & - & 0.9926 & - \\
\hline
\end{tabular}

*It means that it is statistically significant at level $P<0.05$.

Appendix 2 Results of the changes of the TFP and its decomposition index of the individual tertiary public TCMHs from 2010 to 2011 


\begin{tabular}{|c|c|c|c|c|c|c|c|c|c|c|c|c|c|c|}
\hline \multirow{2}{*}{$\begin{array}{l}\text { TCMH } \\
\text { NO }\end{array}$} & \multirow[t]{2}{*}{ TFPC } & \multirow[b]{2}{*}{$\begin{array}{l}\text { Lower } \\
\text { Bounds }\end{array}$} & \multicolumn{3}{|c|}{ TEC } & \multicolumn{3}{|c|}{$\mathrm{TC}$} & \multicolumn{3}{|c|}{ PTEPC } & \multicolumn{3}{|c|}{ SEC } \\
\hline & & & $\begin{array}{l}\text { Upper } \\
\text { Bounds }\end{array}$ & & $\begin{array}{l}\text { Lower } \\
\text { Bounds }\end{array}$ & $\begin{array}{l}\text { Upper } \\
\text { Bounds }\end{array}$ & & $\begin{array}{l}\text { Lower } \\
\text { Bounds }\end{array}$ & $\begin{array}{l}\text { Upper } \\
\text { Bounds }\end{array}$ & & $\begin{array}{l}\text { Lower } \\
\text { Bounds }\end{array}$ & $\begin{array}{l}\text { Upper } \\
\text { Bounds }\end{array}$ & & $\begin{array}{l}\mathrm{LC} \\
\mathrm{Br}\end{array}$ \\
\hline T1 & 1.0161 & 0.9426 & 1.0317 & 1.0462 & 0.9307 & 1.1472 & 0.9712 & 0.8558 & 1.0450 & 1.0393 & 0.8809 & 1.1616 & 1.0066 & 0. \\
\hline T2 & $0.7934^{*}$ & 0.7661 & 0.8050 & $0.8204^{*}$ & 0.7423 & 0.9064 & 0.9671 & 0.8509 & 1.0440 & $0.7241^{*}$ & 0.6171 & 0.8820 & 1.1329 & 0 . \\
\hline T3 & $1.1544^{*}$ & 1.1307 & 1.1601 & 1.1050 & 0.9768 & 1.1981 & 1.0447 & 0.9394 & 1.1470 & 1.0640 & 0.8596 & 1.1655 & 1.0385 & 0. \\
\hline T4 & $1.1923^{*}$ & 1.1501 & 1.2003 & 1.1404 & 0.9810 & 1.2729 & 1.0456 & 0.8942 & 1.1629 & 1.1380 & 0.9779 & 1.2756 & 1.0021 & 0. \\
\hline T5 & $1.1492^{*}$ & 1.0910 & 1.1538 & 1.0799 & 0.9070 & 1.2007 & 1.0642 & 0.9087 & 1.1918 & 1.0609 & 0.8741 & 1.1684 & 1.0179 & 0. \\
\hline T6 & $1.0827 *$ & 1.0737 & 1.0915 & 1.0558 & 0.9586 & 1.1286 & 1.0255 & 0.9503 & 1.1109 & 1.0566 & 0.9465 & 1.1344 & 0.9992 & 0. \\
\hline T7 & $0.8299 *$ & 0.8070 & 0.9414 & $0.7846^{*}$ & 0.7263 & 0.9234 & 1.0577 & 0.9489 & 1.1594 & $0.7689 *$ & 0.7010 & 0.9159 & 1.0203 & 0. \\
\hline T8 & $1.0443^{*}$ & 1.0343 & 1.0502 & 1.0450 & 0.9614 & 1.1285 & 0.9993 & 0.9135 & 1.0712 & 1.0218 & 0.9291 & 1.0858 & 1.0227 & 1. \\
\hline T9 & $1.1142^{*}$ & 1.0553 & 1.1149 & $1.1826 *$ & 1.0008 & 1.3338 & 0.9422 & 0.7840 & 1.0510 & 1.1800 & 0.9990 & 1.3253 & 1.0022 & 0. \\
\hline T10 & $0.9352^{*}$ & 0.8553 & 0.9369 & 0.9553 & 0.8221 & 1.0599 & 0.9790 & 0.8190 & 1.0590 & 0.9473 & 0.8093 & 1.0458 & 1.0084 & 0. \\
\hline T11 & $1.0328 *$ & 1.0265 & 1.0361 & 0.9942 & 0.8756 & 1.1209 & 1.0389 & 0.8871 & 1.1468 & 1.0000 & 0.5441 & 1.2857 & 0.9942 & 0. \\
\hline T12 & $1.0994^{*}$ & 1.0978 & 1.1127 & 1.0776 & 0.9933 & 1.1754 & 1.0202 & 0.9227 & 1.0974 & 1.0000 & 0.8171 & 1.0863 & 1.0776 & 0. \\
\hline T13 & $1.1209 *$ & 1.1099 & 1.1412 & 1.1159* & 1.0332 & 1.2039 & 1.0045 & 0.9219 & 1.0753 & 1.0000 & 0.5396 & 1.3344 & 1.1159 & 0. \\
\hline T14 & $1.1449 *$ & 1.1318 & 1.1663 & $1.1289 *$ & 1.0406 & 1.2131 & 1.0142 & 0.9363 & 1.0911 & 1.1361 & 0.9712 & 1.3008 & 0.9937 & 0. \\
\hline T15 & $1.0854^{*}$ & 1.0827 & 1.1103 & 1.0550 & 0.9584 & 1.1709 & 1.0288 & 0.9154 & 1.1224 & 1.0387 & 0.8621 & 1.2095 & 1.0157 & 0. \\
\hline T16 & $1.1188^{*}$ & 1.1163 & 1.1257 & $1.1475^{\star}$ & 1.0380 & 1.2805 & 0.9750 & 0.8502 & 1.0599 & 1.0971 & 0.9819 & 1.1871 & 1.0459 & 0. \\
\hline T17 & $1.1928 *$ & 1.1716 & 1.2201 & $1.2113^{*}$ & 1.0850 & 1.3323 & 0.9847 & 0.8748 & 1.0689 & 1.1717 & 1.0245 & 1.2596 & $1.0339 *$ & 1. \\
\hline T18 & $0.8437 *$ & 0.8126 & 0.8631 & $0.8385^{*}$ & 0.7521 & 0.9047 & 1.0062 & 0.9149 & 1.1015 & $0.7494^{*}$ & 0.6851 & 0.8181 & 1.1188 & 0. \\
\hline T19 & $1.0553^{*}$ & 1.0521 & 1.0735 & 1.0741 & 0.9904 & 1.1850 & 0.9825 & 0.8805 & 1.0583 & 1.0305 & 0.9243 & 1.0959 & $1.0424^{\star}$ & 1. \\
\hline T20 & $1.2668^{*}$ & 1.2648 & 1.4506 & $1.4388 *$ & 1.0337 & 1.8464 & 0.8804 & 0.6379 & 1.1064 & $1.4653^{*}$ & 1.0834 & 1.8950 & 0.9820 & 0. \\
\hline T21 & $0.9881 *$ & 0.7578 & 0.9838 & 1.0267 & 0.7904 & 1.0739 & 0.9624 & 0.8250 & 1.0239 & 1.0674 & 0.8874 & 1.1458 & 0.9618 & 0. \\
\hline T22 & $0.8884 *$ & 0.8286 & 0.8945 & $0.9143^{*}$ & 0.8123 & 0.9877 & 0.9717 & 0.8661 & 1.0471 & 0.9528 & 0.6292 & 1.1115 & 0.9596 & 0 . \\
\hline T23 & $1.2838 *$ & 1.2599 & 1.3234 & $1.3445^{\star}$ & 1.1993 & 1.5188 & 0.9548 & 0.8248 & 1.0462 & $1.3348 *$ & 1.1482 & 1.5256 & 1.0073 & 0 . \\
\hline T24 & $1.1681^{*}$ & 1.1679 & 1.1829 & $1.2613^{*}$ & 1.0552 & 1.4916 & 0.9261 & 0.7224 & 1.0599 & $1.2285^{*}$ & 1.0173 & 1.4154 & 1.0267 & 0. \\
\hline T25 & $1.0879 *$ & 1.0704 & 1.1043 & $1.1122^{*}$ & 1.0092 & 1.2249 & 0.9782 & 0.8696 & 1.0562 & 1.0804 & 0.9588 & 1.1638 & $1.0294^{*}$ & 1. \\
\hline T26 & $1.0548 *$ & 1.0405 & 1.0631 & 1.0529 & 0.9593 & 1.1431 & 1.0019 & 0.9065 & 1.0840 & 1.0125 & 0.9018 & 1.0695 & $1.0398 *$ & 1. \\
\hline T27 & $1.2993^{*}$ & 1.1694 & 1.2991 & $1.3741^{*}$ & 1.1376 & 1.5252 & 0.9456 & 0.7843 & 1.0483 & $1.5006 *$ & 1.2815 & 1.7512 & 0.9157 & 0. \\
\hline T28 & $0.9697 *$ & 0.9401 & 0.9738 & 1.0424 & 0.8662 & 1.2202 & 0.9303 & 0.7395 & 1.0558 & 1.0412 & 0.7210 & 1.2516 & 1.0011 & 0. \\
\hline T29 & $0.7607^{*}$ & 0.6433 & 0.7681 & $0.7851 *$ & 0.6525 & 0.8390 & 0.9689 & 0.8582 & 1.0389 & $0.7010^{*}$ & 0.5597 & 0.7401 & $1.1200 *$ & 1. \\
\hline T30 & $0.9803^{*}$ & 0.9393 & 0.9898 & 0.9937 & 0.8997 & 1.0718 & 0.9865 & 0.8865 & 1.0609 & 0.9732 & 0.8349 & 1.0316 & $1.0211^{*}$ & 1. \\
\hline Т31 & $1.0946 *$ & 1.0872 & 1.1311 & $1.2605^{\star}$ & 1.0145 & 1.5420 & 0.8684 & 0.6299 & 1.0168 & $1.2857^{*}$ & 1.0872 & 1.5849 & 0.9804 & 0 . \\
\hline T32 & $1.2834^{*}$ & 1.2470 & 1.2850 & $1.3527 *$ & 1.1785 & 1.5117 & 0.9487 & 0.8073 & 1.0464 & $1.3389 *$ & 1.1388 & 1.4848 & 1.0103 & 0. \\
\hline T33 & $1.0859 *$ & 1.0659 & 1.1438 & $1.0805^{\star}$ & 1.0068 & 1.2007 & 1.0050 & 0.9100 & 1.0814 & 0.8908 & 0.7824 & 1.0560 & 1.2130 & 0. \\
\hline T34 & $0.9645^{\star}$ & 0.9632 & 0.9654 & $0.9336 *$ & 0.8393 & 0.9957 & 1.0331 & 0.9584 & 1.1271 & $0.8739 *$ & 0.7648 & 0.9212 & $1.0684^{\star}$ & 1. \\
\hline T35 & $0.8530 *$ & 0.7402 & 0.8903 & $0.8095^{*}$ & 0.6408 & 0.8878 & 1.0537 & 0.9310 & 1.1644 & 0.9046 & 0.6686 & 1.0616 & 0.8949 & 0. \\
\hline T36 & $0.8609 *$ & 0.8359 & 0.8697 & $0.8685^{*}$ & 0.7931 & 0.9441 & 0.9913 & 0.8938 & 1.0671 & $0.8372^{*}$ & 0.7493 & 0.8858 & $1.0374^{*}$ & 1. \\
\hline T37 & $1.2356^{*}$ & 1.2341 & 1.2410 & $1.3247 *$ & 1.1677 & 1.5245 & 0.9327 & 0.7669 & 1.0325 & 1.2880 * & 1.1097 & 1.4494 & 1.0284 & 0. \\
\hline T38 & 0.9787 & 0.9762 & 1.0263 & 1.0469 & 0.9325 & 1.2223 & 0.9349 & 0.7740 & 1.0317 & 1.0000 & 0.4869 & 1.3306 & 1.0469 & 0. \\
\hline Т39 & 1.0705 & 0.8733 & 1.0745 & 1.0826 & 0.8845 & 1.1416 & 0.9888 & 0.8819 & 1.0526 & 1.0780 & 0.8675 & 1.1742 & 1.0043 & 0. \\
\hline T40 & $1.2728^{*}$ & 1.2582 & 1.2902 & $1.3185^{*}$ & 1.1943 & 1.4753 & 0.9654 & 0.8320 & 1.0455 & $1.2291^{*}$ & 1.0787 & 1.3222 & $1.0727^{*}$ & 1. \\
\hline T41 & $1.0550 *$ & 1.0432 & 1.0555 & 1.1045 & 0.9891 & 1.2409 & 0.9552 & 0.8143 & 1.0434 & 1.0924 & 0.9812 & 1.1968 & 1.0110 & 0. \\
\hline
\end{tabular}




\begin{tabular}{|c|c|c|c|c|c|c|c|c|c|c|c|c|c|c|}
\hline T42 & 0.5180 * & 0.4263 & 0.5188 & $0.5066^{*}$ & 0.4044 & 0.5219 & 1.0225 & 0.9311 & 1.0957 & $0.3812^{*}$ & 0.3494 & 0.6235 & 1.3290 & -1 \\
\hline T43 & $1.2156^{*}$ & 1.1486 & 1.2272 & $1.3052^{*}$ & 1.1227 & 1.4875 & 0.9314 & 0.7668 & 1.0328 & 1.2906 & 0.9655 & 1.4545 & 1.0113 & 0 . \\
\hline T44 & $1.1276 *$ & 1.1073 & 1.1385 & $1.1567^{*}$ & 1.0521 & 1.2649 & 0.9748 & 0.8695 & 1.0507 & 1.1296* & 1.0212 & 1.2074 & 1.0240 & 0 . \\
\hline T45 & $0.9938 *$ & 0.9545 & 0.9996 & 1.0040 & 0.9136 & 1.0836 & 0.9898 & 0.8961 & 1.0618 & 0.9932 & 0.8928 & 1.0704 & 1.0110 & 0 . \\
\hline T46 & $1.2867 *$ & 1.2696 & 1.3385 & $1.3030 *$ & 1.1819 & 1.5229 & 0.9875 & 0.8092 & 1.0821 & $1.2447^{*}$ & 1.0732 & 1.4250 & 1.0468 & 0 . \\
\hline T47 & 0.9911 & 0.9464 & 1.0331 & 1.0133 & 0.9043 & 1.1580 & 0.9780 & 0.8023 & 1.0671 & 1.0334 & 0.9411 & 1.2064 & 0.9806 & 0 . \\
\hline T48 & 1.0690 * & 1.0575 & 1.0757 & 1.0290 & 0.8738 & 1.1036 & 1.0388 & 0.9546 & 1.1671 & 0.9574 & 0.7227 & 1.0270 & 1.0748 & 0 . \\
\hline T49 & $1.1252^{*}$ & 1.1125 & 1.1453 & $1.1129 *$ & 1.0185 & 1.1966 & 1.0110 & 0.9285 & 1.0891 & 1.0592 & 0.9328 & 1.1166 & $1.0507^{*}$ & 1. \\
\hline T50 & $1.0642^{\star}$ & 1.0442 & 1.1294 & 1.0000 & 0.8181 & 1.1946 & 1.0642 & 0.8452 & 1.2339 & 1.0000 & 0.8030 & 1.2170 & 1.0000 & 0 . \\
\hline T51 & $1.0882^{*}$ & 1.0833 & 1.1052 & 1.0442 & 0.9273 & 1.1564 & 1.0422 & 0.9213 & 1.1523 & 1.0275 & 0.8810 & 1.1621 & 1.0163 & 0 . \\
\hline T52 & $1.0751^{*}$ & 1.0577 & 1.0773 & 1.0523 & 0.9587 & 1.1354 & 1.0217 & 0.9262 & 1.0998 & 1.0375 & 0.8651 & 1.1584 & 1.0143 & 0 . \\
\hline T53 & $1.0976 *$ & 1.0970 & 1.0997 & 1.0573 & 0.9309 & 1.2146 & 1.0382 & 0.8575 & 1.1496 & 1.0000 & 0.8280 & 1.1175 & 1.0573 & 1. \\
\hline T54 & $1.0615^{\star}$ & 1.0440 & 1.0640 & 0.9834 & 0.7509 & 1.1635 & 1.0794 & 0.8255 & 1.2775 & 0.9829 & 0.7564 & 1.1713 & 1.0005 & 0 . \\
\hline T55 & 0.9828 & 0.9467 & 1.0271 & 1.0720 & 0.9021 & 1.2699 & 0.9168 & 0.7281 & 1.0409 & 0.9594 & 0.8162 & 1.1314 & 1.1174 & 0 . \\
\hline T56 & $0.9763^{*}$ & 0.9629 & 0.9803 & 1.0070 & 0.9138 & 1.1332 & 0.9696 & 0.8267 & 1.0508 & 0.9936 & 0.8829 & 1.1464 & 1.0134 & 0 . \\
\hline T57 & $1.3523^{*}$ & 1.2296 & 1.4382 & 1.2520 & 0.9636 & 1.4702 & 1.0801 & 0.8714 & 1.2541 & 1.2526 & 0.9394 & 1.4818 & 0.9995 & 0 . \\
\hline T58 & $1.1255^{*}$ & 1.1209 & 1.1294 & 1.0769 & 0.9144 & 1.1581 & 1.0452 & 0.9602 & 1.1814 & 1.0000 & 0.7895 & 1.0573 & $1.0769 *$ & 1. \\
\hline T59 & $1.1150 *$ & 1.0462 & 1.1179 & 1.0540 & 0.8673 & 1.1775 & 1.0578 & 0.8903 & 1.1985 & 1.0429 & 0.8344 & 1.1717 & 1.0106 & 0 . \\
\hline T60 & $1.1586 *$ & 1.1579 & 1.1962 & $1.1371^{*}$ & 1.0468 & 1.3030 & 1.0189 & 0.8662 & 1.1085 & 1.0877 & 0.8977 & 1.2572 & 1.0454 & 0. \\
\hline T61 & $0.9794^{*}$ & 0.9739 & 0.9836 & 0.9384 & 0.8477 & 1.0024 & 1.0436 & 0.9653 & 1.1345 & 0.9061 & 0.6951 & 1.0051 & 1.0357 & 0. \\
\hline T62 & $0.9336 *$ & 0.9235 & 0.9341 & 0.9372 & 0.8364 & 1.0241 & 0.9961 & 0.8871 & 1.0891 & $0.8371 *$ & 0.6977 & 0.9058 & 1.1196 & 0 . \\
\hline T63 & $0.8617^{*}$ & 0.8596 & 0.9113 & 1.0000 & 0.6981 & 1.2700 & 0.8617 & 0.5900 & 1.0664 & 1.0000 & 0.6207 & 1.2715 & 1.0000 & 0. \\
\hline T64 & $1.0628 *$ & 1.0473 & 1.1049 & 1.0727 & 0.9732 & 1.1830 & 0.9908 & 0.8922 & 1.0681 & 1.0218 & 0.9056 & 1.1004 & $1.0498 *$ & 1. \\
\hline T65 & $0.9856 *$ & 0.8714 & 0.9930 & 1.0323 & 0.8560 & 1.1389 & 0.9547 & 0.7835 & 1.0367 & 1.0795 & 0.8562 & 1.2193 & 0.9563 & 0. \\
\hline T66 & $1.0965^{*}$ & 1.0760 & 1.0973 & 1.1071 & 0.9777 & 1.2751 & 0.9904 & 0.8089 & 1.0873 & 1.0633 & 0.8997 & 1.2086 & 1.0412 & 0 . \\
\hline T67 & $1.0511^{*}$ & 1.0060 & 1.0770 & 1.0783 & 0.9520 & 1.2582 & 0.9748 & 0.7917 & 1.0659 & 1.0834 & 0.9660 & 1.2723 & 0.9953 & 0 . \\
\hline T68 & $1.0742^{*}$ & 1.0312 & 1.0796 & 1.0814 & 0.9592 & 1.2043 & 0.9933 & 0.8492 & 1.0744 & 1.0816 & 0.9047 & 1.2655 & 0.9998 & 0 . \\
\hline T69 & 1.1198* & 1.0806 & 1.1267 & 1.0000 & 0.6927 & 1.1666 & 1.1198 & 0.8747 & 1.3611 & 1.0000 & 0.7000 & 1.1845 & 1.0000 & 0. \\
\hline T70 & $1.0436 *$ & 1.0194 & 1.0637 & 1.0068 & 0.8889 & 1.1213 & 1.0365 & 0.9011 & 1.1420 & 0.9981 & 0.8707 & 1.1093 & 1.0088 & 0. \\
\hline T71 & $1.0632 *$ & 1.0091 & 1.1047 & 0.9926 & 0.8057 & 1.1372 & 1.0710 & 0.8872 & 1.2205 & 0.9754 & 0.7766 & 1.1041 & 1.0177 & 0. \\
\hline T72 & $1.1077^{*}$ & 1.0912 & 1.1178 & $1.1026 *$ & 1.0127 & 1.1887 & 1.0046 & 0.9175 & 1.0770 & 1.0897 & 0.7694 & 1.3351 & 1.0119 & 0. \\
\hline T73 & 0.9451 * & 0.8753 & 0.9490 & $0.9335^{*}$ & 0.8257 & 0.9897 & 1.0125 & 0.9190 & 1.0883 & 0.9518 & 0.8055 & 1.0455 & 0.9807 & 0 . \\
\hline T74 & 1.0607 & 0.7424 & 1.0481 & 1.1121 & 0.7848 & 1.1479 & 0.9537 & 0.8287 & 1.0285 & 1.2094 & 0.9562 & 1.2764 & $0.9196 *$ & 0 . \\
\hline $\begin{array}{l}\text { Geo- } \\
\text { mean }\end{array}$ & 1.0484 & - & - & 1.0553 & - & - & 0.9935 & - & - & 1.0274 & - & - & 1.0272 & - \\
\hline
\end{tabular}

*It means that it is statistically significant at level $P<0.05$.

Appendix 3 Results of the changes of the TFP and its decomposition index of the individual tertiary public TCMHs from 2011 to 2012 


\begin{tabular}{|c|c|c|c|c|c|c|c|c|c|c|c|c|c|c|}
\hline \multirow{2}{*}{$\begin{array}{l}\text { TCMH } \\
\text { NO }\end{array}$} & \multirow[t]{2}{*}{ TFPC } & \multirow[b]{2}{*}{$\begin{array}{l}\text { Lower } \\
\text { Bounds }\end{array}$} & \multicolumn{3}{|c|}{ TEC } & \multicolumn{3}{|c|}{$\mathrm{TC}$} & \multicolumn{3}{|c|}{ PTEPC } & \multicolumn{3}{|c|}{ SEC } \\
\hline & & & $\begin{array}{l}\text { Upper } \\
\text { Bounds }\end{array}$ & & $\begin{array}{l}\text { Lower } \\
\text { Bounds }\end{array}$ & $\begin{array}{l}\text { Upper } \\
\text { Bounds }\end{array}$ & & $\begin{array}{l}\text { Lower } \\
\text { Bounds }\end{array}$ & $\begin{array}{l}\text { Upper } \\
\text { Bounds }\end{array}$ & & $\begin{array}{l}\text { Lower } \\
\text { Bounds }\end{array}$ & $\begin{array}{l}\text { Upper } \\
\text { Bounds }\end{array}$ & & $\mathrm{L}$ \\
\hline T1 & $1.0531 *$ & 1.0357 & 1.0726 & 0.9740 & 0.9103 & 1.0326 & $1.0812^{*}$ & 1.0116 & 1.1486 & 0.9775 & 0.9019 & 1.0603 & 0.9963 & 0 \\
\hline T2 & 0.9806 & 0.9088 & 1.0081 & $0.8741^{*}$ & 0.7624 & 0.9099 & 1.1219* & 1.0785 & 1.2106 & 0.9522 & 0.8719 & 1.0856 & $0.9180 *$ & 0 \\
\hline T3 & $1.1318^{*}$ & 1.0956 & 1.1884 & 1.0543 & 0.9639 & 1.1451 & 1.0735 & 0.9948 & 1.1542 & 1.0506 & 0.9470 & 1.1408 & 1.0035 & 0 \\
\hline T4 & $1.1117^{*}$ & 1.1014 & 1.1231 & 1.0320 & 0.9427 & 1.1178 & 1.0773 & 0.9776 & 1.1627 & 1.0432 & 0.9598 & 1.1382 & 0.9893 & 0 \\
\hline T5 & $0.9128 *$ & 0.8967 & 0.9939 & 0.8199* & 0.7594 & 0.9026 & $1.1132 *$ & 1.0547 & 1.2008 & 0.8540 * & 0.7804 & 0.9602 & 0.9602 & 0 \\
\hline T6 & $1.2610 *$ & 1.2515 & 1.2679 & $1.1651 *$ & 1.0710 & 1.2652 & 1.0823 & 0.9785 & 1.1608 & $1.1691 *$ & 1.0762 & 1.2656 & 0.9966 & 0 \\
\hline T7 & $1.0674^{*}$ & 1.0373 & 1.0866 & $0.9433^{*}$ & 0.8372 & 0.9963 & $1.1315^{\star}$ & 1.0627 & 1.2389 & 0.9610 & 0.8684 & 1.0233 & 0.9816 & 0 \\
\hline T8 & $1.1255^{*}$ & 1.1034 & 1.1384 & 1.0503 & 0.9475 & 1.1242 & 1.0716 & 0.9787 & 1.1621 & 1.0326 & 0.9299 & 1.1016 & 1.0171 & 0 \\
\hline T9 & $1.0643^{*}$ & 1.0567 & 1.0758 & 1.0060 & 0.9417 & 1.0857 & 1.0579 & 0.9662 & 1.1194 & 1.0048 & 0.9371 & 1.0874 & 1.0012 & 0 \\
\hline T10 & $1.1012^{\star}$ & 1.0863 & 1.1259 & 1.0398 & 0.9565 & 1.1453 & 1.0590 & 0.9487 & 1.1386 & 1.0000 & 0.7721 & 1.2384 & 1.0398 & 0 \\
\hline T11 & $1.0281^{*}$ & 1.0067 & 1.0388 & $0.9075^{\star}$ & 0.8009 & 0.9634 & 1.1329* & 1.0531 & 1.2412 & 1.0000 & 0.7526 & 1.2615 & 0.9075 & 0 \\
\hline T12 & $1.1283^{*}$ & 1.1209 & 1.1629 & 1.0072 & 0.9171 & 1.0777 & $1.1201^{*}$ & 1.0397 & 1.2220 & 0.9952 & 0.8870 & 1.1219 & 1.0121 & 0 \\
\hline T13 & $1.0979 *$ & 1.0773 & 1.1196 & 0.9901 & 0.8734 & 1.0802 & 1.1089* & 1.0020 & 1.2180 & 0.9785 & 0.8578 & 1.1043 & 1.0119 & 0 \\
\hline T14 & $1.1300 *$ & 1.1023 & 1.1536 & 1.0084 & 0.8927 & 1.0622 & $1.1205^{*}$ & 1.0465 & 1.2349 & 1.0481 & 0.9539 & 1.1296 & $0.9622^{*}$ & 0 \\
\hline T15 & $1.0559 *$ & 1.0219 & 1.1384 & 0.9436 & 0.8640 & 1.0192 & 1.1190* & 1.0794 & 1.2047 & 0.9502 & 0.8755 & 1.0408 & 0.9931 & 0 \\
\hline T16 & 1.0111 & 0.9953 & 1.0138 & $0.8891 *$ & 0.7812 & 0.9319 & $1.1373^{\star}$ & 1.0768 & 1.2543 & $0.9255^{\star}$ & 0.8424 & 0.9775 & $0.9607 *$ & 0 \\
\hline T17 & $1.1246^{*}$ & 1.0798 & 1.1931 & 1.0205 & 0.9506 & 1.0860 & 1.1019* & 1.0548 & 1.1761 & 0.9903 & 0.8990 & 1.0349 & $1.0305^{*}$ & 1 \\
\hline T18 & $1.0809 *$ & 1.0640 & 1.0956 & $0.9589 *$ & 0.8670 & 0.9935 & $1.1273^{*}$ & 1.0856 & 1.2248 & 0.9523 & 0.8644 & 1.0181 & 1.0069 & 0 \\
\hline T19 & $1.0078 *$ & 1.0016 & 1.0468 & 0.9590 & 0.8749 & 1.0558 & 1.0509 & 0.9371 & 1.1418 & 0.9519 & 0.8387 & 1.0296 & 1.0075 & 0 \\
\hline T20 & $1.0381 *$ & 1.0172 & 1.0510 & $0.9157 *$ & 0.8109 & 0.9622 & $1.1337^{*}$ & 1.0699 & 1.2442 & $0.9357^{*}$ & 0.8489 & 0.9918 & $0.9786 *$ & 0 \\
\hline T21 & $0.9527 *$ & 0.9196 & 0.9778 & $0.8509 *$ & 0.7564 & 0.8948 & 1.1196* & 1.0443 & 1.2198 & $0.8604^{*}$ & 0.7733 & 0.9141 & 0.9889 & 0 \\
\hline T22 & $0.9271 *$ & 0.8934 & 0.9820 & 0.8620 * & 0.7929 & 0.9474 & $1.0756 *$ & 1.0089 & 1.1437 & 0.9545 & 0.9185 & 1.1349 & $0.9031 *$ & 0 \\
\hline T23 & $1.2646 *$ & 1.2391 & 1.2867 & $1.1567 *$ & 1.0468 & 1.2259 & $1.0933^{*}$ & 1.0297 & 1.1797 & $1.2700 *$ & 1.1924 & 1.3989 & $0.9107 *$ & 0 \\
\hline T24 & $1.1112^{*}$ & 1.0880 & 1.1221 & 0.9822 & 0.8769 & 1.0196 & $1.1313^{*}$ & 1.0882 & 1.2344 & 0.9885 & 0.9019 & 1.0579 & 0.9936 & 0 \\
\hline T25 & $1.0491 *$ & 1.0361 & 1.0500 & 1.0113 & 0.9193 & 1.1084 & 1.0374 & 0.9243 & 1.1226 & 1.0000 & 0.7721 & 1.1049 & 1.0113 & 0 \\
\hline T26 & $0.8386 *$ & 0.8032 & 0.8604 & $0.7823^{*}$ & 0.7204 & 0.8275 & 1.0721 & 0.9951 & 1.1455 & $0.8024^{*}$ & 0.6636 & 0.8603 & 0.9749 & 0 \\
\hline T27 & $0.9636 *$ & 0.9512 & 0.9990 & $0.8683^{*}$ & 0.8047 & 0.9188 & 1.1098* & 1.0534 & 1.1908 & $0.8757 *$ & 0.7953 & 0.9389 & 0.9915 & 0 \\
\hline T28 & $1.1273^{*}$ & 1.1260 & 1.1640 & 1.0343 & 0.9492 & 1.1490 & 1.0899 & 0.9820 & 1.1854 & 1.0350 & 0.9609 & 1.1554 & 0.9993 & 0 \\
\hline T29 & $1.0633^{*}$ & 1.0433 & 1.1045 & 1.0432 & 0.9661 & 1.1797 & 1.0193 & 0.8861 & 1.0992 & 0.9844 & 0.8472 & 1.1296 & 1.0598 & 0 \\
\hline T30 & 0.9768 & 0.9338 & 1.0298 & $0.8845^{*}$ & 0.8153 & 0.9334 & $1.1043^{*}$ & 1.0613 & 1.1823 & $0.8932^{*}$ & 0.8129 & 0.9448 & 0.9903 & 0 \\
\hline Т31 & $1.2615^{\star}$ & 1.2599 & 1.2654 & $1.1256 *$ & 1.0049 & 1.1755 & $1.1207^{*}$ & 1.0701 & 1.2303 & 1.2390 * & 1.1488 & 1.3391 & $0.9085^{*}$ & 0 \\
\hline T32 & $1.0480 *$ & 1.0451 & 1.0510 & $0.9306 *$ & 0.8425 & 0.9644 & $1.1261^{*}$ & 1.0837 & 1.2235 & 1.0000 & 0.7575 & 1.2759 & 0.9306 & 0 \\
\hline T33 & $0.7882^{*}$ & 0.7508 & 0.8160 & $0.7192^{*}$ & 0.6500 & 0.7620 & 1.0960 * & 1.0429 & 1.1673 & $0.8072^{*}$ & 0.6941 & 0.8826 & $0.8910 *$ & 0 \\
\hline T34 & 1.1219* & 1.0633 & 1.2027 & 1.0033 & 0.9007 & 1.0724 & $1.1182^{*}$ & 1.0847 & 1.2106 & 1.0000 & 0.8966 & 1.0824 & 1.0033 & 0 \\
\hline T35 & $1.1102^{*}$ & 1.0614 & 1.1383 & 0.9834 & 0.8737 & 1.0178 & $1.1289 *$ & 1.0913 & 1.2259 & $1.1341^{*}$ & 1.0403 & 1.2004 & $0.8671 *$ & 0 \\
\hline T36 & $1.4685^{*}$ & 1.4326 & 1.5566 & $1.3444^{*}$ & 1.2539 & 1.4558 & $1.0923 *$ & 1.0282 & 1.1634 & $1.3842^{*}$ & 1.2911 & 1.5327 & 0.9713 & 0 \\
\hline T37 & $1.0110 *$ & 1.0089 & 1.0131 & $0.9134^{\star}$ & 0.8134 & 0.9653 & 1.1069* & 1.0405 & 1.2158 & 0.9486 & 0.8611 & 1.0838 & 0.9628 & 0 \\
\hline T38 & $1.1664^{*}$ & 1.1614 & 1.1790 & 1.0324 & 0.9341 & 1.0710 & $1.1298 *$ & 1.0890 & 1.2300 & $1.0861 *$ & 1.0214 & 1.1454 & $0.9505^{\star}$ & 0 \\
\hline Т39 & 1.0650 * & 1.0583 & 1.0917 & 0.9553 & 0.8801 & 1.0067 & $1.1148 *$ & 1.0626 & 1.2014 & 0.9617 & 0.8896 & 1.0202 & 0.9934 & 0 \\
\hline T40 & $1.1071 *$ & 1.0575 & 1.1266 & 1.0389 & 0.9449 & 1.1130 & 1.0656 & 0.9706 & 1.1438 & 1.0528 & 0.9680 & 1.1374 & 0.9868 & 0 \\
\hline T41 & $1.1392^{*}$ & 1.1028 & 1.1726 & $1.0887 *$ & 1.0185 & 1.1799 & 1.0463 & 0.9484 & 1.1074 & $1.0889 *$ & 1.0130 & 1.1896 & 0.9998 & 0 \\
\hline
\end{tabular}




\begin{tabular}{|c|c|c|c|c|c|c|c|c|c|c|c|c|c|c|}
\hline T42 & $1.1608 *$ & 1.1350 & 1.1741 & 1.0544 & 0.9416 & 1.1261 & $1.1009 *$ & 1.0054 & 1.1981 & 1.0732 & 0.9739 & 1.1544 & 0.9825 & 0 \\
\hline T43 & $1.0343^{*}$ & 1.0269 & 1.0427 & 1.0000 & 0.8488 & 1.1124 & 1.0343 & 0.9066 & 1.1695 & 1.0000 & 0.8128 & 1.1241 & 1.0000 & 0 \\
\hline T44 & $1.0262^{\star}$ & 1.0178 & 1.0368 & 0.9465 & 0.8587 & 1.0365 & 1.0842 & 0.9757 & 1.1779 & 0.9546 & 0.8742 & 1.0618 & 0.9915 & 0 \\
\hline T45 & 1.1319* & 1.1235 & 1.1363 & 1.0000 & 0.8723 & 1.0916 & 1.1319* & 1.0208 & 1.2563 & 1.0000 & 0.8751 & 1.1278 & 1.0000 & 0 \\
\hline T46 & $1.0136 *$ & 1.0111 & 1.0210 & 1.0000 & 0.9105 & 1.1323 & 1.0136 & 0.8631 & 1.0964 & 1.0000 & 0.9062 & 1.1365 & 1.0000 & 0 \\
\hline T47 & $1.1629 *$ & 1.1576 & 1.2299 & 1.0969 & 0.9739 & 1.2444 & 1.0602 & 0.9252 & 1.1795 & 1.0971 & 0.9623 & 1.2539 & 0.9998 & 0 \\
\hline T48 & $0.9809 *$ & 0.9638 & 0.9913 & 0.9491 & 0.8738 & 1.0496 & 1.0335 & 0.9188 & 1.1086 & 0.9829 & 0.8753 & 1.1069 & 0.9657 & 0 \\
\hline T49 & $1.1068 *$ & 1.0283 & 1.1391 & 0.9952 & 0.8756 & 1.0433 & $1.1121 *$ & 1.0637 & 1.1918 & 1.0570 & 0.9830 & 1.1754 & $0.9415^{*}$ & 0 \\
\hline T50 & $1.0357^{*}$ & 1.0263 & 1.0433 & 1.0000 & 0.8833 & 1.1463 & 1.0357 & 0.8559 & 1.1376 & 1.0000 & 0.8586 & 1.1595 & 1.0000 & 0 \\
\hline T51 & 1.0882 & 0.9784 & 1.1454 & 1.0532 & 0.8693 & 1.1711 & 1.0332 & 0.9063 & 1.1576 & 1.0871 & 0.9173 & 1.2286 & 0.9689 & 0 \\
\hline T52 & $1.0312^{\star}$ & 1.0102 & 1.0881 & 0.9709 & 0.8944 & 1.0655 & 1.0621 & 0.9800 & 1.1528 & 0.9823 & 0.8984 & 1.0799 & 0.9885 & 0 \\
\hline T53 & 1.0089 & 0.9696 & 1.0319 & 0.9379 & 0.8395 & 1.0276 & 1.0757 & 0.9661 & 1.1569 & 0.9202 & 0.8030 & 1.0249 & 1.0193 & 0 \\
\hline T54 & 0.9930 & 0.9657 & 1.0048 & $0.8993^{*}$ & 0.8054 & 0.9638 & $1.1043^{*}$ & 1.0052 & 1.2025 & 0.9192 & 0.7792 & 1.0215 & 0.9783 & 0 \\
\hline T55 & $0.9237 *$ & 0.8909 & 0.9361 & $0.8395^{\star}$ & 0.7311 & 0.8875 & $1.1003^{*}$ & 1.0077 & 1.2035 & $0.8876^{*}$ & 0.7800 & 0.9719 & 0.9458 & 0 \\
\hline T56 & $1.0531 *$ & 1.0332 & 1.0708 & 1.0061 & 0.9361 & 1.0998 & 1.0468 & 0.9446 & 1.1099 & 1.0000 & 0.9228 & 1.1529 & 1.0061 & 0 \\
\hline T57 & $1.0540 *$ & 1.0455 & 1.0572 & 0.9807 & 0.8960 & 1.0586 & 1.0748 & 0.9815 & 1.1566 & 0.9792 & 0.8894 & 1.0667 & 1.0016 & 0 \\
\hline T58 & $1.2785^{\star}$ & 1.2656 & 1.3068 & $1.1960 *$ & 1.1208 & 1.2863 & 1.0690 & 0.9890 & 1.1347 & $1.1946 *$ & 1.1121 & 1.2843 & 1.0012 & 0 \\
\hline T59 & 1.0130 & 0.9757 & 1.0195 & $0.9175^{*}$ & 0.8134 & 0.9774 & 1.1040 * & 1.0226 & 1.1964 & 0.9350 & 0.8297 & 1.0364 & 0.9813 & 0 \\
\hline T60 & $1.0983^{*}$ & 1.0640 & 1.1214 & 1.0780 & 0.9824 & 1.2082 & 1.0188 & 0.8859 & 1.1087 & 1.0564 & 0.9333 & 1.1701 & 1.0204 & 0 \\
\hline T61 & $1.0549 *$ & 1.0433 & 1.0599 & 1.0000 & 0.8415 & 1.1431 & 1.0549 & 0.8774 & 1.1920 & 1.0000 & 0.8238 & 1.1514 & 1.0000 & 0 \\
\hline T62 & $1.0378 *$ & 1.0105 & 1.0511 & 0.9685 & 0.8762 & 1.0510 & 1.0715 & 0.9693 & 1.1573 & 0.9430 & 0.8264 & 1.0075 & $1.0271 *$ & 1 \\
\hline T63 & $1.1265^{\star}$ & 1.0634 & 1.1388 & 1.0592 & 0.9296 & 1.1566 & 1.0635 & 0.9363 & 1.1549 & 1.0727 & 0.9536 & 1.1920 & 0.9874 & 0 \\
\hline T64 & 1.2797 * & 1.2610 & 1.3313 & $1.1418^{*}$ & 1.0328 & 1.2268 & $1.1207^{*}$ & 1.0403 & 1.2225 & $1.4351^{*}$ & 1.1715 & 1.6721 & $0.7956^{*}$ & 0 \\
\hline T65 & 1.0556 * & 1.0215 & 1.1131 & 0.9968 & 0.9304 & 1.1082 & 1.0590 & 0.9743 & 1.1126 & 0.9970 & 0.9272 & 1.1229 & 0.9998 & 0 \\
\hline $\begin{array}{l}\text { Geo- } \\
\text { mean }\end{array}$ & 1.0692 & - & - & 0.9836 & - & - & 1.0869 & - & - & 1.0053 & - & - & 0.9785 & - \\
\hline
\end{tabular}

*It means that it is statistically significant at level $P<0.05$.

Appendix 4 Results of the changes of the TFP and its decomposition index of the individual tertiary public TCMHs from 2012 to 2014 


\begin{tabular}{|c|c|c|c|c|c|c|c|c|c|c|c|c|c|c|}
\hline \multirow{2}{*}{$\begin{array}{l}\text { TCMH } \\
\text { NO }\end{array}$} & \multirow[t]{2}{*}{ TFPC } & \multirow[b]{2}{*}{$\begin{array}{l}\text { Lower } \\
\text { Bounds }\end{array}$} & \multicolumn{3}{|c|}{ TEC } & \multicolumn{3}{|c|}{$\mathrm{TC}$} & \multicolumn{3}{|c|}{ PTEPC } & \multicolumn{3}{|c|}{ SEC } \\
\hline & & & $\begin{array}{l}\text { Upper } \\
\text { Bounds }\end{array}$ & & $\begin{array}{l}\text { Lower } \\
\text { Bounds }\end{array}$ & $\begin{array}{l}\text { Upper } \\
\text { Bounds }\end{array}$ & & $\begin{array}{l}\text { Lower } \\
\text { Bounds }\end{array}$ & $\begin{array}{l}\text { Upper } \\
\text { Bounds }\end{array}$ & & $\begin{array}{l}\text { Lower } \\
\text { Bounds }\end{array}$ & $\begin{array}{l}\text { Upper } \\
\text { Bounds }\end{array}$ & & $\mathrm{L}$ \\
\hline T1 & $1.1475^{\star}$ & 1.1222 & 1.1949 & 1.0567 & 0.8699 & 1.2146 & 1.0860 & 0.9187 & 1.2518 & 1.0651 & 0.8599 & 1.3063 & 0.9921 & 0 \\
\hline T2 & 1.0726 & 0.9020 & 1.1327 & 0.9916 & 0.7963 & 1.0941 & 1.0816 & 0.9093 & 1.2030 & 0.8859 & 0.7891 & 1.0524 & 1.1194 & 0 \\
\hline T3 & $1.1153^{*}$ & 1.1023 & 1.1243 & 1.0187 & 0.7883 & 1.2006 & 1.0949 & 0.8557 & 1.2954 & 1.0107 & 0.7614 & 1.2045 & 1.0079 & 0 \\
\hline T4 & $1.0805^{\star}$ & 1.0469 & 1.0975 & 0.9427 & 0.6697 & 1.0973 & 1.1462 & 0.9176 & 1.3959 & 0.9948 & 0.7593 & 1.1970 & $0.9476 *$ & 0 \\
\hline T5 & $1.0994^{*}$ & 1.0362 & 1.1192 & 0.9720 & 0.7753 & 1.0505 & $1.1311^{*}$ & 1.0156 & 1.3049 & 1.0574 & 0.7840 & 1.2714 & 0.9192 & 0 \\
\hline T6 & $0.9855^{*}$ & 0.9741 & 0.9917 & $0.8224 *$ & 0.6092 & 0.9112 & $1.1982^{*}$ & 1.0488 & 1.4423 & $0.8563^{*}$ & 0.6769 & 0.9817 & $0.9605^{*}$ & 0 \\
\hline T7 & $5.0263^{*}$ & 4.8202 & 5.2149 & 4.2080 * & 3.4479 & 4.5150 & $1.1944^{*}$ & 1.0956 & 1.3868 & $4.4281^{*}$ & 3.3479 & 6.1973 & 0.9503 & 0 \\
\hline T8 & $1.0451 *$ & 1.0359 & 1.0888 & $0.8279 *$ & 0.6151 & 0.8995 & $1.2623^{*}$ & 1.1574 & 1.5162 & $0.8354^{\star}$ & 0.6355 & 0.9131 & 0.9910 & 0 \\
\hline T9 & 1.1309 & 0.8093 & 1.1316 & 1.1807 & 0.8231 & 1.4057 & 0.9579 & 0.5504 & 1.0917 & 1.1911 & 0.8352 & 1.4383 & 0.9912 & 0 \\
\hline T10 & $1.1543^{*}$ & 1.1274 & 1.1699 & 1.1434 & 0.9647 & 1.3582 & 1.0095 & 0.7742 & 1.1380 & 1.1689 & 0.9821 & 1.4095 & 0.9782 & 0 \\
\hline T11 & $1.0798 *$ & 1.0110 & 1.1276 & 0.9925 & 0.7994 & 1.1259 & 1.0880 & 0.9226 & 1.2443 & 1.0000 & 0.4119 & 1.4104 & 0.9925 & 0 \\
\hline T12 & 0.7630 * & 0.7112 & 0.8228 & 0.6480 * & 0.5298 & 0.7043 & $1.1774^{*}$ & 1.0800 & 1.3701 & 0.7337 & 0.5831 & 1.2016 & 0.8832 & -( \\
\hline T13 & $1.1383^{*}$ & 1.1310 & 1.1672 & 0.9220 * & 0.7129 & 0.9993 & $1.2346^{*}$ & 1.1303 & 1.4657 & 0.9931 & 0.8142 & 1.1822 & 0.9284 & 0 \\
\hline T14 & $1.0591 *$ & 1.0581 & 1.0794 & $0.8899 *$ & 0.7035 & 0.9773 & $1.1901^{*}$ & 1.0637 & 1.3968 & 0.9314 & 0.7732 & 1.1213 & 0.9555 & 0 \\
\hline T15 & $0.8923 *$ & 0.8259 & 0.9482 & $0.7987 *$ & 0.6853 & 0.8860 & 1.1172 & 0.9817 & 1.2620 & $0.8028 *$ & 0.7202 & 0.9074 & 0.9949 & 0 \\
\hline T16 & $1.7046^{*}$ & 1.6706 & 1.7822 & $1.5859 *$ & 1.3859 & 1.8092 & 1.0748 & 0.9180 & 1.1986 & $1.6265^{\star}$ & 1.4559 & 1.9221 & $0.9751 *$ & 0 \\
\hline T17 & $1.1141 *$ & 1.1009 & 1.1254 & 0.9528 & 0.7829 & 1.0371 & $1.1693^{*}$ & 1.0550 & 1.3509 & 1.0014 & 0.8606 & 1.1006 & $0.9515^{*}$ & 0 \\
\hline T18 & $1.1911^{*}$ & 1.1664 & 1.2276 & 1.1134 & 0.9238 & 1.2832 & 1.0698 & 0.8923 & 1.2253 & 1.1551 & 0.9908 & 1.3344 & 0.9639 & 0 \\
\hline T19 & $0.8814 *$ & 0.7640 & 0.9028 & $0.8418 *$ & 0.6870 & 0.9446 & 1.0469 & 0.8500 & 1.1590 & $0.8454^{*}$ & 0.6295 & 0.9596 & 0.9957 & 0 \\
\hline T20 & $1.0142^{*}$ & 1.0136 & 1.1010 & 1.0828 & 0.8847 & 1.4997 & 0.9366 & 0.4341 & 1.0994 & 1.0320 & 0.8313 & 1.4254 & 1.0493 & 0 \\
\hline T21 & $0.7449 *$ & 0.5686 & 0.7650 & $0.6751 *$ & 0.4926 & 0.7216 & 1.1033 & 0.9424 & 1.2388 & $0.6742^{*}$ & 0.4810 & 0.7286 & 1.0013 & 0 \\
\hline T22 & $1.0751 *$ & 1.0605 & 1.1041 & $0.8783^{*}$ & 0.6966 & 0.9501 & $1.2241^{*}$ & 1.1211 & 1.4477 & $0.8959 *$ & 0.7313 & 0.9803 & 0.9804 & 0 \\
\hline T23 & $1.1912^{\star}$ & 1.1366 & 1.2155 & 1.0459 & 0.8469 & 1.1360 & $1.1390 *$ & 1.0325 & 1.3004 & 1.1094 & 0.9194 & 1.2166 & 0.9428 & 0 \\
\hline T24 & $1.3530 *$ & 1.3343 & 1.4028 & $1.2818 *$ & 1.1150 & 1.4828 & 1.0555 & 0.8841 & 1.1816 & $1.3748 *$ & 1.2474 & 1.6106 & 0.9324 & 0 \\
\hline T25 & $1.1883^{*}$ & 1.1974 & 1.5016 & $1.1126^{*}$ & 1.0400 & 1.4885 & 1.0680 & 0.8663 & 1.2012 & 0.8992 & 0.8199 & 1.1639 & $1.2373^{*}$ & 1 \\
\hline T26 & $1.1980 *$ & 1.1730 & 1.2215 & 1.0356 & 0.8362 & 1.1345 & 1.1569* & 1.0373 & 1.3291 & 1.0540 & 0.8684 & 1.2017 & 0.9825 & 0 \\
\hline T27 & $1.0782^{*}$ & 1.0661 & 1.0837 & 1.0085 & 0.8527 & 1.1366 & 1.0691 & 0.9098 & 1.2106 & 1.1060 & 0.9614 & 1.3044 & 0.9119 & 0 \\
\hline T28 & $1.0822^{\star}$ & 1.0799 & 1.0908 & $0.8677^{*}$ & 0.6609 & 0.9409 & $1.2472^{*}$ & 1.1334 & 1.4900 & 0.9241 & 0.7659 & 1.0188 & $0.9389 *$ & 0 \\
\hline T29 & $1.0965^{\star}$ & 1.0817 & 1.1052 & 1.1161 & 0.9701 & 1.3356 & 0.9824 & 0.7418 & 1.0933 & 1.0806 & 0.6794 & 1.4208 & 1.0329 & 0 \\
\hline Т30 & 1.0641 & 0.9598 & 1.0721 & 1.0427 & 0.8378 & 1.2229 & 1.0205 & 0.7526 & 1.1474 & 1.0450 & 0.7965 & 1.2496 & 0.9979 & 0 \\
\hline Т31 & $0.9335^{*}$ & 0.6400 & 0.9378 & 0.9471 & 0.6634 & 1.0764 & 0.9856 & 0.6735 & 1.1047 & 0.9533 & 0.6368 & 1.0887 & 0.9935 & 0 \\
\hline T32 & $1.1985^{*}$ & 1.1263 & 1.2162 & 1.1491 & 0.9799 & 1.3077 & 1.0429 & 0.8488 & 1.1611 & 1.0000 & 0.3192 & 1.4869 & 1.1491 & 0 \\
\hline T33 & $1.0815^{\star}$ & 1.0698 & 1.0990 & 0.9572 & 0.8088 & 1.0427 & 1.1299* & 1.0181 & 1.2863 & 1.0603 & 0.8442 & 1.3693 & 0.9027 & 0 \\
\hline T34 & $1.2321^{*}$ & 1.2189 & 1.2929 & $1.1864^{\star}$ & 1.0408 & 1.4016 & 1.0385 & 0.8389 & 1.1594 & $1.1986 *$ & 1.0654 & 1.4373 & 0.9898 & 0 \\
\hline T35 & $0.9446 *$ & 0.9093 & 0.9627 & $0.8120 *$ & 0.6358 & 0.8854 & $1.1633^{*}$ & 1.0448 & 1.3450 & 0.9694 & 0.8076 & 1.0799 & $0.8376 *$ & 0 \\
\hline T36 & $1.3477 *$ & 1.3398 & 1.4257 & $1.2016 *$ & 1.0176 & 1.3441 & $1.1216 *$ & 1.0000 & 1.2903 & $1.2618 *$ & 1.0852 & 1.4425 & 0.9523 & 0 \\
\hline T37 & $0.7063^{*}$ & 0.6470 & 0.7099 & $0.7053^{*}$ & 0.5832 & 0.8375 & 1.0015 & 0.7187 & 1.1130 & $0.6919 *$ & 0.6107 & 0.8729 & 1.0193 & 0 \\
\hline T38 & $1.1863^{*}$ & 1.1730 & 1.2042 & 1.0658 & 0.9074 & 1.1816 & 1.1131 & 0.9785 & 1.2556 & $1.1320 *$ & 1.0076 & 1.2580 & 0.9415 & 0 \\
\hline Т39 & $0.9130 *$ & 0.8584 & 0.9924 & $0.7788 *$ & 0.6173 & 0.8749 & $1.1723^{*}$ & 1.0759 & 1.3566 & $0.7622^{*}$ & 0.5747 & 0.8487 & 1.0218 & 0 \\
\hline T40 & 1.0338 & 0.9786 & 1.0818 & 1.0298 & 0.8945 & 1.2230 & 1.0039 & 0.7797 & 1.1148 & 0.9777 & 0.7943 & 1.1310 & $1.0533^{*}$ & 1 \\
\hline T41 & $1.1756 *$ & 1.1287 & 1.1911 & 1.1554 & 0.9648 & 1.3873 & 1.0175 & 0.7497 & 1.1467 & 1.2000 * & 1.0048 & 1.4482 & 0.9628 & 0 \\
\hline
\end{tabular}




\begin{tabular}{|c|c|c|c|c|c|c|c|c|c|c|c|c|c|c|}
\hline T42 & 0.9959 & 0.9478 & 1.0082 & 0.9493 & 0.8133 & 1.0771 & 1.0492 & 0.8680 & 1.1673 & 1.0301 & 0.8340 & 1.2541 & 0.9215 & 0 \\
\hline T43 & $1.3504^{*}$ & 1.3408 & 1.3532 & 1.1154 & 0.8766 & 1.2171 & $1.2107^{*}$ & 1.0910 & 1.4262 & $1.2943^{*}$ & 1.1364 & 1.4896 & 0.8617 & 0 \\
\hline T44 & 0.7768 * & 0.6846 & 0.8167 & $0.7170 *$ & 0.5970 & 0.7988 & 1.0834 & 0.9203 & 1.2095 & $0.7288^{*}$ & 0.6040 & 0.8290 & 0.9839 & 0 \\
\hline T45 & $1.0847 *$ & 1.0664 & 1.1413 & $0.8771^{*}$ & 0.6954 & 0.9523 & $1.2367^{*}$ & 1.1299 & 1.4699 & 0.9112 & 0.7794 & 1.0141 & 0.9625 & 0 \\
\hline T46 & 0.9796 & 0.9490 & 1.0311 & 0.9850 & 0.8034 & 1.2459 & 0.9946 & 0.6786 & 1.1474 & 1.0000 & 0.7688 & 1.2949 & 0.9850 & 0 \\
\hline T47 & 1.0187 & 0.9672 & 1.0506 & 0.9837 & 0.8108 & 1.1283 & 1.0355 & 0.8409 & 1.1736 & 1.0172 & 0.8173 & 1.2163 & 0.9671 & 0 \\
\hline T48 & 1.1969* & 1.1109 & 1.2448 & 1.0000 & 0.7323 & 1.1060 & $1.1969 *$ & 1.0851 & 1.4028 & 1.0000 & 0.7124 & 1.1897 & 1.0000 & 0 \\
\hline T49 & $1.1941 *$ & 1.1907 & 1.2188 & 1.0730 & 0.7554 & 1.3260 & 1.1128 & 0.7887 & 1.3730 & 1.1194 & 0.8288 & 1.4305 & $0.9585^{*}$ & 0 \\
\hline T50 & $1.3120 *$ & 1.3114 & 1.4607 & $1.2667^{*}$ & 1.1509 & 1.5684 & 1.0357 & 0.8145 & 1.1616 & $1.3300 *$ & 1.2390 & 1.7026 & 0.9524 & 0 \\
\hline T51 & $1.1436 *$ & 1.0248 & 1.1918 & 1.0298 & 0.8519 & 1.1201 & 1.1105 & 0.9796 & 1.2530 & 1.0876 & 0.9355 & 1.1997 & 0.9469 & 0 \\
\hline T52 & 0.6690 * & 0.6529 & 0.6892 & 0.5660 * & 0.4717 & 0.6238 & 1.1820 * & 1.0605 & 1.3713 & 0.5790 * & 0.5191 & 0.6854 & $0.9775^{\star}$ & 0 \\
\hline T53 & $1.1104^{*}$ & 1.0630 & 1.1523 & 1.0715 & 0.8090 & 1.3470 & 1.0363 & 0.6912 & 1.2325 & 1.1624 & 0.8626 & 1.4979 & 0.9219 & 0 \\
\hline T54 & $1.1576 *$ & 1.0885 & 1.1625 & 1.0822 & 0.8221 & 1.2717 & 1.0697 & 0.8047 & 1.2543 & 1.1113 & 0.8433 & 1.3296 & 0.9738 & 0 \\
\hline T55 & $0.9831 *$ & 0.9772 & 0.9890 & 0.9274 & 0.7782 & 1.0530 & 1.0601 & 0.8934 & 1.2053 & 0.9494 & 0.6871 & 1.1785 & 0.9768 & 0 \\
\hline T56 & 1.0148 & 0.9777 & 1.0395 & $0.8248 *$ & 0.6438 & 0.8865 & $1.2304^{*}$ & 1.1252 & 1.4599 & 1.2392 & 0.8700 & 1.4859 & 0.6655 & 0 \\
\hline T57 & $1.3100 *$ & 1.2349 & 1.3985 & $1.1533^{*}$ & 1.0010 & 1.2704 & 1.1359* & 1.0098 & 1.2918 & 1.1379* & 1.0545 & 1.3695 & 1.0135 & 0 \\
\hline T58 & 1.0683 & 0.8892 & 1.0791 & 1.0000 & 0.4100 & 1.3583 & 1.0683 & 0.4512 & 1.3818 & 1.0000 & 0.3727 & 1.3967 & 1.0000 & 0 \\
\hline T59 & $1.1531 *$ & 1.1174 & 1.2306 & 1.1336 & 0.9584 & 1.3647 & 1.0172 & 0.7982 & 1.1449 & 1.0750 & 0.9032 & 1.2955 & 1.0546 & 0 \\
\hline T60 & $0.8956 *$ & 0.8528 & 0.9755 & 0.8528 & 0.7491 & 1.0078 & 1.0501 & 0.8885 & 1.1633 & $0.8030 *$ & 0.6162 & 0.9382 & 1.0621 & 0 \\
\hline T61 & $1.1096 *$ & 1.0773 & 1.1484 & 1.0468 & 0.8834 & 1.2054 & 1.0600 & 0.8864 & 1.1986 & 1.0900 & 0.9440 & 1.2718 & $0.9604^{*}$ & 0 \\
\hline T62 & $1.1178 *$ & 1.0250 & 1.1723 & 0.9817 & 0.7600 & 1.0775 & $1.1386 *$ & 1.0452 & 1.3083 & 1.0325 & 0.7962 & 1.2770 & 0.9509 & 0 \\
\hline T63 & 1.0659 & 0.9984 & 1.1543 & 1.0025 & 0.8350 & 1.1824 & 1.0632 & 0.8843 & 1.2070 & 1.1342 & 0.9710 & 1.4384 & 0.8839 * & 0 \\
\hline T64 & $1.0671^{*}$ & 1.0479 & 1.0754 & 1.0000 & 0.6528 & 1.2840 & 1.0671 & 0.6440 & 1.3298 & 1.0000 & 0.6325 & 1.2990 & 1.0000 & 0 \\
\hline T65 & $1.1973^{*}$ & 1.1141 & 1.2067 & 1.0855 & 0.6743 & 1.3206 & 1.1030 & 0.7501 & 1.3643 & 1.1088 & 0.7140 & 1.3733 & 0.9790 & 0 \\
\hline T66 & $1.0565^{\star}$ & 1.0465 & 1.0702 & 1.0101 & 0.8289 & 1.1842 & 1.0460 & 0.8353 & 1.2063 & 1.0112 & 0.8219 & 1.2015 & 0.9989 & 0 \\
\hline T67 & 1.0836 & 0.9905 & 1.1640 & 0.9443 & 0.7379 & 1.0509 & $1.1475^{*}$ & 1.0529 & 1.3116 & 0.8653 & 0.6629 & 1.2174 & 1.0913 & 0 \\
\hline T68 & $0.8802^{*}$ & 0.8707 & 0.9425 & $0.7935^{*}$ & 0.6880 & 0.8953 & 1.1092 & 0.9922 & 1.2615 & 0.8968 & 0.6147 & 1.0184 & 0.8849 & 0 \\
\hline $\begin{array}{l}\text { Geo- } \\
\text { mean }\end{array}$ & 1.0939 & - & - & 0.9971 & - & - & 1.0971 & - & - & 1.0053 & - & - & 0.9717 & - \\
\hline
\end{tabular}

*It means that it is statistically significant at level $P<0.05$.

Appendix 5 Results of the changes of the TFP and its decomposition index of the individual tertiary public TCMHs from 2014 to 2015 


\begin{tabular}{|c|c|c|c|c|c|c|c|c|c|c|c|c|c|c|}
\hline \multirow{2}{*}{$\begin{array}{l}\text { TCMH } \\
\text { NO }\end{array}$} & \multirow[t]{2}{*}{ TFPC } & \multirow[b]{2}{*}{$\begin{array}{l}\text { Lower } \\
\text { Bounds }\end{array}$} & \multicolumn{3}{|c|}{ TEC } & \multicolumn{3}{|c|}{$\mathrm{TC}$} & \multicolumn{3}{|c|}{ PTEPC } & \multicolumn{3}{|c|}{ SEC } \\
\hline & & & $\begin{array}{l}\text { Upper } \\
\text { Bounds }\end{array}$ & & $\begin{array}{l}\text { Lower } \\
\text { Bounds }\end{array}$ & $\begin{array}{l}\text { Upper } \\
\text { Bounds }\end{array}$ & & $\begin{array}{l}\text { Lower } \\
\text { Bounds }\end{array}$ & $\begin{array}{l}\text { Upper } \\
\text { Bounds }\end{array}$ & & $\begin{array}{l}\text { Lower } \\
\text { Bounds }\end{array}$ & $\begin{array}{l}\text { Upper } \\
\text { Bounds }\end{array}$ & & $\begin{array}{l}\mathrm{LC} \\
\mathrm{Bc}\end{array}$ \\
\hline T1 & $0.8638 *$ & 0.7935 & 0.9120 & $0.8499 *$ & 0.6807 & 0.9440 & 1.0163 & 0.9090 & 1.1514 & $0.8658 *$ & 0.6736 & 0.9624 & 0.9817 & 0. \\
\hline T2 & $1.2153^{*}$ & 1.2004 & 1.2173 & 1.1860 * & 1.0077 & 1.3120 & 1.0247 & 0.8962 & 1.1549 & 1.1953* & 1.0711 & 1.3154 & 0.9922 & 0. \\
\hline T3 & 1.0280 * & 1.0187 & 1.0502 & 1.0510 & 0.8316 & 1.1926 & 0.9781 & 0.8349 & 1.1533 & 1.0847 & 0.8774 & 1.2481 & 0.9690 & 0. \\
\hline T4 & $1.2120 *$ & 1.1509 & 1.3092 & $1.2066^{*}$ & 1.0188 & 1.3418 & 1.0045 & 0.9179 & 1.1316 & 1.1598* & 1.0194 & 1.3463 & 1.0403 & 0. \\
\hline T5 & $0.9167^{*}$ & 0.8883 & 0.9498 & 0.9456 & 0.8021 & 1.0554 & 0.9695 & 0.8477 & 1.0920 & 0.9794 & 0.8511 & 1.1147 & 0.9655 & 0. \\
\hline T6 & $0.9111 *$ & 0.8898 & 0.9316 & $0.9124 *$ & 0.7841 & 0.9938 & 0.9986 & 0.9019 & 1.1170 & 1.1205 & 0.5073 & 1.3002 & 0.8143 & 0 . \\
\hline T7 & $0.9553^{*}$ & 0.9466 & 0.9693 & 0.9884 & 0.8231 & 1.1115 & 0.9665 & 0.8329 & 1.1039 & 1.0129 & 0.8696 & 1.1529 & $0.9759 *$ & 0. \\
\hline T8 & $0.9844^{*}$ & 0.9450 & 0.9861 & 0.9461 & 0.7403 & 1.0682 & 1.0404 & 0.8760 & 1.2115 & 0.9591 & 0.7499 & 1.0942 & 0.9865 & 0. \\
\hline T9 & $0.8815^{\star}$ & 0.8018 & 0.9273 & $0.8718^{*}$ & 0.7460 & 0.9493 & 1.0111 & 0.9254 & 1.1114 & 0.8590 * & 0.7601 & 0.9141 & 1.0149 & 0. \\
\hline T10 & $0.9421 *$ & 0.9042 & 0.9514 & 0.9255 & 0.7741 & 1.0188 & 1.0180 & 0.8916 & 1.1457 & 0.9124 & 0.7513 & 1.0071 & 1.0143 & 0. \\
\hline T11 & $0.9739 *$ & 0.9262 & 0.9961 & 0.9811 & 0.8111 & 1.0567 & 0.9926 & 0.8946 & 1.1306 & 1.0000 & 0.7039 & 1.2927 & 0.9811 & 0. \\
\hline T12 & 0.9942 & 0.9607 & 1.0014 & 0.9808 & 0.8102 & 1.0562 & 1.0137 & 0.9132 & 1.1590 & 1.0000 & 0.4733 & 1.2203 & 0.9808 & 0. \\
\hline T13 & $1.0281^{*}$ & 1.0245 & 1.0357 & 1.0638 & 0.9031 & 1.1898 & 0.9665 & 0.8372 & 1.0930 & 1.0130 & 0.8577 & 1.1186 & 1.0502 & 0. \\
\hline T14 & $0.7013^{*}$ & 0.6687 & 0.7512 & $0.7110 *$ & 0.6311 & 0.7965 & 0.9863 & 0.8947 & 1.0863 & $0.7383^{*}$ & 0.6159 & 0.8313 & 0.9630 & 0. \\
\hline T15 & $0.8936 *$ & 0.8844 & 0.9454 & $0.8887 *$ & 0.7492 & 0.9911 & 1.0055 & 0.9125 & 1.1488 & 0.9235 & 0.7622 & 1.0715 & 0.9623 & 0 . \\
\hline T16 & $0.4469 *$ & 0.4102 & 0.4992 & $0.4523^{*}$ & 0.3810 & 0.5230 & 0.9882 & 0.8919 & 1.1054 & $0.3841^{*}$ & 0.3188 & 0.5975 & 1.1774 & -0 \\
\hline T17 & $0.9612^{*}$ & 0.9482 & 0.9773 & 0.9509 & 0.8436 & 1.0286 & 1.0109 & 0.9238 & 1.1142 & 0.9643 & 0.8842 & 1.0384 & 0.9861 & 0. \\
\hline T18 & $1.0240 *$ & 1.0135 & 1.0243 & 1.0194 & 0.9020 & 1.0955 & 1.0046 & 0.9227 & 1.1074 & 1.0383 & 0.9410 & 1.1212 & 0.9817 & 0. \\
\hline T19 & 1.0162 & 0.9872 & 1.0320 & 1.0327 & 0.8912 & 1.1291 & 0.9840 & 0.8752 & 1.0962 & 1.0598 & 0.9370 & 1.1311 & 0.9745 & 0. \\
\hline T20 & $1.0682^{*}$ & 1.0116 & 1.1748 & 1.0624 & 0.9134 & 1.1901 & 1.0054 & 0.9245 & 1.1237 & 1.0285 & 0.9202 & 1.1321 & 1.0330 & 0. \\
\hline T21 & $0.9050 *$ & 0.8917 & 0.9242 & $0.9036 *$ & 0.7990 & 0.9820 & 1.0015 & 0.9123 & 1.1077 & 0.9157 & 0.8095 & 1.0151 & 0.9868 & 0. \\
\hline T22 & 0.9579 & 0.9527 & 1.0099 & 0.9399 & 0.8323 & 1.0476 & 1.0191 & 0.9202 & 1.1416 & 0.9025 & 0.8477 & 1.0412 & 1.0414 & 0. \\
\hline T23 & $0.9086 *$ & 0.9023 & 0.9471 & $0.8945^{\star}$ & 0.7494 & 0.9883 & 1.0157 & 0.9198 & 1.1606 & 0.9408 & 0.8022 & 1.0730 & 0.9508 & 0. \\
\hline T24 & $1.0409 *$ & 1.0244 & 1.0662 & 1.0365 & 0.9012 & 1.1248 & 1.0043 & 0.9204 & 1.1200 & 1.0140 & 0.8604 & 1.1899 & 1.0222 & 0 . \\
\hline T25 & $1.0596 *$ & 1.0588 & 1.0686 & 1.0356 & 0.8886 & 1.1533 & 1.0231 & 0.8978 & 1.1527 & 1.0369 & 0.8813 & 1.1684 & 0.9987 & 0. \\
\hline T26 & $1.0627 *$ & 1.0611 & 1.0984 & 1.0267 & 0.8619 & 1.1615 & 1.0350 & 0.8993 & 1.1923 & 1.0410 & 0.8776 & 1.1891 & 0.9863 & 0. \\
\hline T27 & $1.2214^{*}$ & 1.2258 & 1.3266 & $1.1905^{*}$ & 1.0486 & 1.3596 & 1.0260 & 0.9074 & 1.1654 & 1.1960 * & 1.0544 & 1.3760 & 0.9954 & 0. \\
\hline T28 & $0.9215^{\star}$ & 0.9051 & 0.9232 & 0.9426 & 0.8048 & 1.0395 & 0.9777 & 0.8571 & 1.0979 & 0.9729 & 0.8523 & 1.0714 & 0.9688 & 0. \\
\hline T29 & $0.8028 *$ & 0.6241 & 0.8677 & $0.8035^{*}$ & 0.5855 & 0.8809 & 0.9992 & 0.8976 & 1.0977 & $0.7087^{*}$ & 0.4980 & 0.7572 & 1.1337 & 0. \\
\hline T30 & $1.0643^{*}$ & 1.0539 & 1.0792 & 1.0540 & 0.9267 & 1.1399 & 1.0098 & 0.9256 & 1.1153 & $1.1191^{*}$ & 1.0415 & 1.2270 & 0.9418 & 0 . \\
\hline Т31 & $1.0521^{*}$ & 1.0456 & 1.0530 & 1.0574 & 0.9184 & 1.1519 & 0.9949 & 0.8940 & 1.1097 & $1.1234^{*}$ & 1.0067 & 1.1917 & 0.9413 & 0. \\
\hline T32 & $0.9175^{*}$ & 0.9063 & 0.9397 & 0.9224 & 0.8057 & 1.0174 & 0.9947 & 0.8909 & 1.1106 & 0.9179 & 0.7946 & 1.0072 & 1.0049 & 0. \\
\hline T33 & 0.9918 & 0.9822 & 1.0658 & 0.9901 & 0.8860 & 1.1512 & 1.0017 & 0.8571 & 1.1188 & 0.9350 & 0.7107 & 1.1215 & 1.0590 & 0. \\
\hline T34 & $1.0410 *$ & 1.0307 & 1.0450 & 1.0243 & 0.8677 & 1.1110 & 1.0164 & 0.9190 & 1.1485 & 0.9812 & 0.8338 & 1.0742 & 1.0439 & 0. \\
\hline T35 & $1.0455^{*}$ & 1.0455 & 1.0568 & 1.0818 & 0.9155 & 1.2128 & 0.9665 & 0.8372 & 1.0967 & $1.1805^{*}$ & 1.0740 & 1.3480 & $0.9164^{*}$ & 0 . \\
\hline T36 & $1.0134 *$ & 1.0074 & 1.0141 & 1.0094 & 0.8430 & 1.1138 & 1.0039 & 0.8873 & 1.1443 & 1.0377 & 0.8162 & 1.2485 & 0.9728 & 0. \\
\hline T37 & $1.1247^{*}$ & 1.1232 & 1.1946 & 1.0972 & 0.9525 & 1.2471 & 1.0251 & 0.9046 & 1.1625 & 1.0978 & 0.9534 & 1.2557 & 0.9995 & 0. \\
\hline T38 & $0.9722^{*}$ & 0.9474 & 0.9734 & 0.9369 & 0.7438 & 1.0472 & 1.0377 & 0.8877 & 1.2058 & 0.9262 & 0.7298 & 1.0695 & 1.0116 & 0 . \\
\hline Т39 & $0.9087 *$ & 0.9068 & 0.9126 & 0.8919* & 0.7775 & 0.9799 & 1.0188 & 0.9064 & 1.1346 & 0.8962 & 0.7256 & 1.0529 & 0.9952 & 0 . \\
\hline T40 & $1.0610 *$ & 1.0603 & 1.0774 & 1.0545 & 0.9274 & 1.1416 & 1.0062 & 0.9209 & 1.1177 & 1.0171 & 0.8610 & 1.1799 & 1.0368 & 0. \\
\hline T41 & $0.9370 *$ & 0.8984 & 0.9542 & 0.9232 & 0.7985 & 1.0004 & 1.0149 & 0.9190 & 1.1216 & $0.9070 *$ & 0.7691 & 0.9737 & 1.0179 & 0 . \\
\hline
\end{tabular}




\begin{tabular}{|c|c|c|c|c|c|c|c|c|c|c|c|c|c|c|}
\hline T42 & 1.0050 & 0.9999 & 1.0660 & 1.0101 & 0.8440 & 1.2016 & 0.9949 & 0.8023 & 1.1498 & 0.9203 & 0.7423 & 1.0951 & 1.0976 & 0. \\
\hline T43 & 0.9636 & 0.9033 & 1.0475 & 0.9735 & 0.8592 & 1.0941 & 0.9899 & 0.9040 & 1.0893 & 1.0209 & 0.9164 & 1.1273 & 0.9535 & 0. \\
\hline T44 & $1.0830^{*}$ & 1.0774 & 1.1198 & 1.0763 & 0.9414 & 1.1749 & 1.0062 & 0.9200 & 1.1268 & $1.1294^{*}$ & 1.0143 & 1.2230 & 0.9530 & 0. \\
\hline T45 & 1.0759* & 1.0756 & 1.0802 & 1.0487 & 0.8940 & 1.1665 & 1.0260 & 0.8985 & 1.1594 & 1.0758 & 0.9294 & 1.2078 & 0.9748 & 0. \\
\hline T46 & $0.9715^{*}$ & 0.9180 & 0.9948 & 0.9871 & 0.8476 & 1.0720 & 0.9842 & 0.8828 & 1.0884 & 1.0167 & 0.8869 & 1.0764 & 0.9709 & 0. \\
\hline T47 & $1.1401^{*}$ & 1.1387 & 1.1578 & 1.1642 & 0.9921 & 1.3017 & 0.9792 & 0.8538 & 1.1082 & $1.1950^{*}$ & 1.0399 & 1.3514 & $0.9742^{*}$ & 0. \\
\hline T48 & $1.1417^{*}$ & 1.1245 & 1.1485 & 1.1399 & 0.9749 & 1.2638 & 1.0016 & 0.8793 & 1.1245 & $1.1629 *$ & 1.0118 & 1.3042 & 0.9802 & 0. \\
\hline T49 & $1.2504^{*}$ & 1.1980 & 1.3535 & $1.2389 *$ & 1.0916 & 1.4085 & 1.0093 & 0.8896 & 1.1230 & 1.1642 & 0.9477 & 1.2850 & 1.0641 & 1. \\
\hline T50 & $0.9647^{*}$ & 0.9608 & 0.9722 & 0.9498 & 0.8393 & 1.0354 & 1.0156 & 0.9174 & 1.1234 & 0.8866 & 0.6541 & 1.0682 & 1.0713 & 0. \\
\hline T51 & $0.7614^{\star}$ & 0.7349 & 0.7654 & $0.7836 *$ & 0.6587 & 0.8671 & 0.9717 & 0.8511 & 1.0907 & $0.8494^{*}$ & 0.7434 & 0.9244 & 0.9225 & 0. \\
\hline T52 & $0.9627 *$ & 0.9434 & 0.9990 & 0.9467 & 0.8372 & 1.0436 & 1.0169 & 0.9125 & 1.1292 & 0.9588 & 0.8609 & 1.0716 & 0.9874 & 0. \\
\hline T53 & 0.9931 & 0.9666 & 1.0510 & 1.0104 & 0.9015 & 1.1364 & 0.9829 & 0.8813 & 1.0907 & 0.9870 & 0.8863 & 1.1057 & 1.0236 & 0. \\
\hline T54 & $0.9114^{\star}$ & 0.9085 & 0.9116 & 0.9413 & 0.8202 & 1.0488 & 0.9682 & 0.8430 & 1.0783 & 0.9827 & 0.8772 & 1.0783 & 0.9579 & 0. \\
\hline T55 & $1.0180^{*}$ & 1.0137 & 1.0287 & 0.9972 & 0.8653 & 1.1053 & 1.0209 & 0.9007 & 1.1430 & 0.9884 & 0.8234 & 1.0914 & 1.0089 & 0. \\
\hline T56 & $1.0270^{*}$ & 1.0157 & 1.0449 & 1.0153 & 0.7811 & 1.1428 & 1.0116 & 0.8723 & 1.2052 & 1.0000 & 0.6938 & 1.1432 & 1.0153 & 0. \\
\hline T57 & 0.9829 & 0.9599 & 1.0025 & 0.9652 & 0.7867 & 1.0626 & 1.0184 & 0.9080 & 1.1840 & 0.9825 & 0.8209 & 1.0955 & 0.9825 & 0. \\
\hline T58 & $1.0469 *$ & 1.0224 & 1.0741 & 1.0319 & 0.8642 & 1.1413 & 1.0145 & 0.9109 & 1.1640 & 1.0307 & 0.8598 & 1.1414 & 1.0011 & 0. \\
\hline T59 & 0.9868 & 0.9698 & 1.0117 & 1.0000 & 0.8199 & 1.1308 & 0.9868 & 0.8516 & 1.1349 & 1.0000 & 0.8005 & 1.1455 & 1.0000 & 0. \\
\hline T60 & $1.0314^{*}$ & 1.0191 & 1.1024 & 1.0513 & 0.8345 & 1.2175 & 0.9810 & 0.8245 & 1.1699 & 1.0784 & 0.8765 & 1.2553 & 0.9749 & 0. \\
\hline T61 & $1.1225^{\star}$ & 1.1162 & 1.1315 & 1.1099 & 0.9819 & 1.1999 & 1.0113 & 0.9231 & 1.1169 & $1.1549 *$ & 1.0166 & 1.2833 & 0.9611 & 0 : \\
\hline T62 & $1.1556^{*}$ & 1.1446 & 1.1996 & $1.1405^{*}$ & 1.0172 & 1.2490 & 1.0133 & 0.9249 & 1.1212 & $1.1920^{*}$ & 1.1237 & 1.3219 & 0.9568 & 0. \\
\hline T63 & $0.7855^{\star}$ & 0.7714 & 0.7859 & $0.7722^{\star}$ & 0.6610 & 0.8369 & 1.0172 & 0.9199 & 1.1384 & 0.7179* & 0.6554 & 0.7969 & 1.0756 & 0. \\
\hline T64 & $0.9222^{\star}$ & 0.8974 & 0.9315 & 0.9380 & 0.8019 & 1.0257 & 0.9832 & 0.8750 & 1.1001 & 0.9508 & 0.8192 & 1.0416 & 0.9865 & 0. \\
\hline T65 & $1.0205^{*}$ & 1.0079 & 1.0321 & 1.0341 & 0.7850 & 1.1844 & 0.9868 & 0.8265 & 1.1825 & 1.1394 & 0.8605 & 1.3085 & 0.9076 & 0 . \\
\hline T66 & $0.8764^{*}$ & 0.8350 & 0.9274 & 0.8890 & 0.7161 & 1.0051 & 0.9859 & 0.8554 & 1.1585 & 0.9465 & 0.7616 & 1.0909 & 0.9392 & 0. \\
\hline T67 & $0.6879 *$ & 0.6675 & 0.7054 & $0.6761^{*}$ & 0.5658 & 0.7310 & 1.0175 & 0.9293 & 1.1658 & $0.7479 *$ & 0.4411 & 0.8416 & 0.9039 & 0. \\
\hline T68 & $1.0420^{*}$ & 1.0287 & 1.1163 & 1.0309 & 0.8735 & 1.1413 & 1.0108 & 0.9205 & 1.1538 & 0.9598 & 0.7821 & 1.1130 & 1.0740 & 0. \\
\hline T69 & $1.1287^{*}$ & 1.0883 & 1.1586 & 1.1246 & 0.9822 & 1.2133 & 1.0036 & 0.9211 & 1.1081 & $1.1624^{*}$ & 1.0579 & 1.2542 & 0.9675 & 0. \\
\hline T70 & $1.0232^{*}$ & 1.0135 & 1.0318 & 1.0000 & 0.6685 & 1.2205 & 1.0232 & 0.7415 & 1.2722 & 1.0000 & 0.6504 & 1.2267 & 1.0000 & 0. \\
\hline T71 & 0.9898 & 0.9757 & 1.0370 & 0.9998 & 0.8425 & 1.1171 & 0.9900 & 0.8656 & 1.1338 & 1.0353 & 0.9153 & 1.1466 & 0.9657 & 0 \\
\hline T72 & $0.9600 *$ & 0.9452 & 0.9669 & 0.9578 & 0.8169 & 1.0541 & 1.0022 & 0.8863 & 1.1276 & 0.9732 & 0.8511 & 1.0853 & 0.9842 & $0 . '$ \\
\hline T73 & $0.9591^{*}$ & 0.9136 & 0.9874 & 0.9443 & 0.7843 & 1.0164 & 1.0157 & 0.9223 & 1.1551 & 0.9599 & 0.7762 & 1.0655 & 0.9837 & 0. \\
\hline T74 & $0.9169 *$ & 0.8920 & 0.9264 & 0.9028 & 0.7437 & 0.9818 & 1.0156 & 0.9165 & 1.1600 & $0.9092^{*}$ & 0.7775 & 0.9805 & 0.9929 & 0. \\
\hline T75 & $0.8624^{\star}$ & 0.8458 & 0.8933 & $0.8600^{*}$ & 0.7289 & 0.9518 & 1.0027 & 0.8889 & 1.1396 & $0.8789 *$ & 0.7301 & 0.9909 & 0.9784 & 0. \\
\hline T76 & $0.9393^{*}$ & 0.9020 & 0.9741 & 0.9302 & 0.7647 & 1.0049 & 1.0097 & 0.9290 & 1.1608 & 0.9696 & 0.8197 & 1.0706 & 0.9594 & 0. \\
\hline T77 & $0.9839 *$ & 0.9667 & 0.9909 & 1.0000 & 0.6999 & 1.1748 & 0.9839 & 0.7746 & 1.2066 & 1.0000 & 0.6860 & 1.1724 & 1.0000 & 0. \\
\hline T78 & 1.0017 & 0.9876 & 1.0073 & 0.9838 & 0.7768 & 1.0853 & 1.0182 & 0.9012 & 1.1918 & 0.9901 & 0.7933 & 1.1007 & 0.9936 & 0. \\
\hline T79 & 0.9886 & 0.9749 & 1.0036 & 1.0033 & 0.7323 & 1.1704 & 0.9854 & 0.7992 & 1.1946 & 1.0060 & 0.7248 & 1.1788 & 0.9973 & 0. \\
\hline T80 & $1.0084^{*}$ & 1.0009 & 1.0357 & 1.0346 & 0.8806 & 1.1584 & 0.9746 & 0.8434 & 1.1052 & 1.0993 & 0.4448 & 1.2365 & 0.9412 & 0 \\
\hline T81 & $0.9901 *$ & 0.9866 & 0.9933 & 0.9835 & 0.8632 & 1.0597 & 1.0067 & 0.9224 & 1.1158 & 0.9772 & 0.7772 & 1.1106 & 1.0064 & 0. \\
\hline $\begin{array}{l}\text { Geo- } \\
\text { mean }\end{array}$ & 0.9756 & - & - & 0.9731 & - & - & 1.0025 & - & - & 0.9821 & - & - & 0.9908 & - \\
\hline
\end{tabular}

*It means that it is statistically significant at level $P<0.05$. 


\begin{tabular}{|c|c|c|c|c|c|c|c|c|c|c|c|c|c|c|}
\hline \multirow{2}{*}{$\begin{array}{l}\text { TCMH } \\
\text { NO }\end{array}$} & \multirow[t]{2}{*}{ TFPC } & \multirow[b]{2}{*}{$\begin{array}{l}\text { Lower } \\
\text { Bounds }\end{array}$} & \multicolumn{3}{|c|}{ TEC } & \multicolumn{3}{|c|}{$\mathrm{TC}$} & \multicolumn{3}{|c|}{ PTEPC } & \multicolumn{3}{|c|}{ SEC } \\
\hline & & & $\begin{array}{l}\text { Upper } \\
\text { Bounds }\end{array}$ & & $\begin{array}{l}\text { Lower } \\
\text { Bounds }\end{array}$ & $\begin{array}{l}\text { Upper } \\
\text { Bounds }\end{array}$ & & $\begin{array}{l}\text { Lower } \\
\text { Bounds }\end{array}$ & $\begin{array}{l}\text { Upper } \\
\text { Bounds }\end{array}$ & & $\begin{array}{l}\text { Lower } \\
\text { Bounds }\end{array}$ & $\begin{array}{l}\text { Upper } \\
\text { Bounds }\end{array}$ & & $\mathrm{L}$ \\
\hline T1 & $1.1563^{*}$ & 1.1208 & 1.1924 & $1.4677^{*}$ & 1.4057 & 1.7975 & $0.7879 *$ & 0.5667 & 0.8048 & $1.4192 *$ & 1.3115 & 1.7472 & 1.0342 & 0 \\
\hline T2 & $1.1571^{*}$ & 1.1478 & 1.1817 & $1.3144^{*}$ & 1.1260 & 1.4903 & $0.8804^{*}$ & 0.7553 & 0.9921 & $1.3978 *$ & 1.2694 & 1.5653 & 0.9403 & 0 \\
\hline T3 & 1.0042 & 0.9979 & 1.0089 & 0.9753 & 0.8125 & 1.1571 & 1.0296 & 0.7929 & 1.1750 & 0.9604 & 0.7729 & 1.1346 & 1.0155 & 0 \\
\hline T4 & $1.0784 *$ & 1.0610 & 1.0884 & 1.0579 & 0.9204 & 1.2108 & 1.0194 & 0.8472 & 1.1287 & 1.0084 & 0.8345 & 1.1361 & 1.0491 & 0 \\
\hline T5 & $1.1101^{*}$ & 1.0883 & 1.1267 & 1.0742 & 0.9533 & 1.2342 & 1.0335 & 0.8572 & 1.1335 & 1.0865 & 0.9615 & 1.2387 & 0.9886 & 0 \\
\hline T6 & $1.1111^{*}$ & 1.1111 & 1.1549 & 1.0702 & 0.9445 & 1.2371 & 1.0382 & 0.8656 & 1.1601 & 1.0603 & 0.9281 & 1.2149 & 1.0093 & 0 \\
\hline T7 & $0.9675^{\star}$ & 0.9557 & 0.9848 & $1.5004^{*}$ & 1.4200 & 1.9606 & $0.6449 *$ & 0.3671 & 0.6766 & $1.4800 *$ & 1.2777 & 1.8535 & 1.0137 & 0 \\
\hline T8 & $0.9122^{*}$ & 0.8858 & 0.9318 & 1.0026 & 0.8778 & 1.1259 & 0.9099 & 0.7858 & 1.0000 & 1.0018 & 0.9177 & 1.0987 & 1.0008 & 0 \\
\hline T9 & 0.9899 & 0.9475 & 1.0088 & $1.2242^{*}$ & 1.1578 & 1.4822 & $0.8087 *$ & 0.5901 & 0.8286 & $1.2685^{*}$ & 1.1911 & 1.5339 & 0.9651 & 0 \\
\hline T10 & $1.0731^{*}$ & 1.0661 & 1.1172 & 1.0383 & 0.9384 & 1.2003 & 1.0335 & 0.8598 & 1.1304 & 1.0000 & 0.5367 & 1.3123 & 1.0383 & 0 \\
\hline T11 & $0.9338 *$ & 0.9232 & 0.9452 & 1.0436 & 0.9940 & 1.2360 & $0.8949 *$ & 0.6988 & 0.9363 & 1.0000 & 0.2117 & 1.3594 & 1.0436 & 0 \\
\hline T12 & $0.9274^{*}$ & 0.9186 & 0.9278 & 0.8933 & 0.7719 & 1.0145 & 1.0382 & 0.8753 & 1.1624 & 0.9163 & 0.7930 & 1.0480 & 0.9749 & 0 \\
\hline T13 & 0.9964 & 0.9919 & 1.0018 & 1.0007 & 0.8977 & 1.1067 & 0.9958 & 0.8783 & 1.0901 & 0.9237 & 0.7328 & 1.0416 & 1.0833 & 0 \\
\hline T14 & 1.0185 & 0.9885 & 1.0415 & 0.9918 & 0.8487 & 1.1271 & 1.0268 & 0.8682 & 1.1479 & 1.0131 & 0.8374 & 1.1931 & 0.9790 & 0 \\
\hline T15 & $1.3310 *$ & 1.1693 & 1.4710 & $1.2993^{*}$ & 1.0904 & 1.4955 & 1.0244 & 0.8879 & 1.1313 & $1.4369 *$ & 1.2928 & 1.7385 & 0.9043 & 0 \\
\hline T16 & $1.0505^{\star}$ & 1.0325 & 1.0624 & 1.0644 & 0.9375 & 1.1622 & 0.9869 & 0.8847 & 1.0877 & 1.0872 & 0.9920 & 1.1763 & 0.9790 & 0 \\
\hline T17 & $0.9242^{*}$ & 0.8824 & 0.9485 & 0.9322 & 0.8121 & 1.0139 & 0.9914 & 0.8916 & 1.0880 & 0.9491 & 0.8580 & 1.0300 & 0.9823 & 0 \\
\hline T18 & $1.1362^{*}$ & 1.1362 & 1.1467 & 1.0944 & 0.9505 & 1.2408 & 1.0382 & 0.8821 & 1.1607 & 1.0802 & 0.9444 & 1.1866 & 1.0131 & 0 \\
\hline T19 & 0.9527 & 0.8536 & 1.0359 & $1.0839 *$ & 1.0225 & 1.2523 & $0.8789 *$ & 0.7163 & 0.9150 & 1.0039 & 0.9065 & 1.1005 & $1.0797 *$ & 1 \\
\hline T20 & 1.1969* & 1.1800 & 1.2128 & 1.1879* & 1.0526 & 1.3210 & 1.0077 & 0.8796 & 1.1067 & 1.1780 * & 1.0374 & 1.3276 & 1.0084 & 0 \\
\hline T21 & $1.0365^{\star}$ & 1.0172 & 1.0497 & $1.1428 *$ & 1.0628 & 1.2989 & $0.9069 *$ & 0.7686 & 0.9629 & $1.1576 *$ & 1.0695 & 1.2703 & 0.9872 & 0 \\
\hline T22 & $1.0965^{*}$ & 1.0801 & 1.1286 & 1.2020 * & 1.0971 & 1.4071 & $0.9122^{*}$ & 0.7327 & 0.9827 & $1.1603^{*}$ & 1.0042 & 1.3627 & 1.0359 & 0 \\
\hline T23 & $1.1362^{\star}$ & 1.1013 & 1.2016 & $1.1925^{\star}$ & 1.1227 & 1.3476 & 0.9528 & 0.8162 & 1.0182 & 1.0902 & 0.9355 & 1.2677 & 1.0939 & 0 \\
\hline T24 & $1.1363^{*}$ & 1.1216 & 1.1407 & $1.3330 *$ & 1.1711 & 1.5209 & $0.8524^{*}$ & 0.7081 & 0.9404 & $1.3672^{*}$ & 1.2370 & 1.6089 & 0.9750 & 0 \\
\hline T25 & 0.9853 & 0.9836 & 1.0106 & $1.3051^{*}$ & 1.1807 & 1.5847 & $0.7549 *$ & 0.5630 & 0.8219 & $1.3444^{*}$ & 1.1980 & 1.6008 & 0.9708 & 0 \\
\hline T26 & 1.0598* & 1.0553 & 1.0760 & $1.2942^{*}$ & 1.1981 & 1.5240 & $0.8189 *$ & 0.6509 & 0.8758 & $1.3079 *$ & 1.2129 & 1.5382 & 0.9895 & 0 \\
\hline T27 & 1.0329 & 0.9662 & 1.1394 & 1.1020 * & 1.0317 & 1.2826 & $0.9373 *$ & 0.7917 & 0.9909 & 1.0080 & 0.8712 & 1.1266 & $1.0933^{*}$ & 1 \\
\hline T28 & $0.9313^{*}$ & 0.8613 & 0.9810 & 0.9800 & 0.8608 & 1.0810 & 0.9503 & 0.8465 & 1.0325 & 0.9857 & 0.8633 & 1.0878 & 0.9942 & 0 \\
\hline T29 & $1.0437^{*}$ & 1.0402 & 1.0648 & 1.0507 & 0.9470 & 1.1621 & 0.9933 & 0.8872 & 1.0888 & 1.0589 & 0.9760 & 1.1375 & 0.9923 & 0 \\
\hline Т30 & 0.9926 & 0.9764 & 1.0052 & 0.9602 & 0.8252 & 1.0758 & 1.0337 & 0.8862 & 1.1506 & 0.9436 & 0.8317 & 1.0254 & 1.0176 & 0 \\
\hline Т31 & $0.8566^{*}$ & 0.8060 & 0.9138 & $0.8746 *$ & 0.7883 & 0.9782 & 0.9794 & 0.8627 & 1.0579 & $0.8629 *$ & 0.7500 & 0.9586 & 1.0136 & 0 \\
\hline T32 & $0.6615^{*}$ & 0.6016 & 0.6885 & $0.7552^{*}$ & 0.6768 & 0.8916 & $0.8759 *$ & 0.6956 & 0.9226 & $0.5090 *$ & 0.4590 & 0.6611 & 1.4837 & 0 \\
\hline T33 & $1.0986 *$ & 1.0676 & 1.1398 & $1.2274^{*}$ & 1.1643 & 1.4463 & 0.8950 * & 0.7131 & 0.9424 & $1.2171 *$ & 1.1410 & 1.4251 & 1.0085 & 0 \\
\hline T34 & $0.9679 *$ & 0.9559 & 0.9824 & 0.9323 & 0.8129 & 1.0594 & 1.0382 & 0.8770 & 1.1598 & $0.8866 *$ & 0.7670 & 0.9749 & 1.0515 & 0 \\
\hline T35 & $1.0444^{*}$ & 1.0296 & 1.0512 & 1.0177 & 0.8298 & 1.1579 & 1.0263 & 0.8625 & 1.1794 & 1.0000 & 0.7430 & 1.2027 & 1.0177 & 0 \\
\hline T36 & $1.1057 *$ & 1.0872 & 1.1304 & $1.2833^{*}$ & 1.1737 & 1.4879 & $0.8616^{*}$ & 0.7134 & 0.9281 & $1.3177^{*}$ & 1.2356 & 1.5627 & $0.9740 *$ & 0 \\
\hline T37 & $1.0631 *$ & 1.0482 & 1.0640 & $1.5574^{\star}$ & 1.3991 & 1.9484 & $0.6826 *$ & 0.4438 & 0.7459 & 1.7149* & 1.3894 & 2.1294 & 0.9082 & 0 \\
\hline T38 & 0.9839 & 0.9668 & 1.0132 & 1.0283 & 0.8797 & 1.1365 & 0.9568 & 0.8550 & 1.0784 & 1.0944 & 0.9067 & 1.3361 & 0.9396 & 0 \\
\hline Т39 & $1.0731 *$ & 1.0493 & 1.1247 & $1.1355^{\star}$ & 1.0596 & 1.2747 & 0.9450 & 0.8210 & 1.0114 & 1.0587 & 0.8868 & 1.2105 & 1.0726 & 0 \\
\hline T40 & $0.5161^{*}$ & 0.4755 & 0.6060 & $0.6538 *$ & 0.5978 & 0.8649 & $0.7894^{\star}$ & 0.5903 & 0.8168 & $0.5029 *$ & 0.4631 & 0.6625 & $1.3001 *$ & 1 \\
\hline T41 & $1.0845^{*}$ & 1.0633 & 1.1065 & $1.1758 *$ & 1.0871 & 1.3127 & $0.9223^{*}$ & 0.7974 & 0.9898 & $1.2408^{*}$ & 1.0889 & 1.3392 & 0.9476 & 0 \\
\hline
\end{tabular}




\begin{tabular}{|c|c|c|c|c|c|c|c|c|c|c|c|c|c|c|}
\hline 42 & 3094* & 2745 & 1.3595 & $1.3053^{*}$ & 1.1825 & 1.4741 & 1.0032 & 0.8711 & 1.0944 & $1.3450^{*}$ & 1.1933 & 1.4619 & 0.9705 & 0 \\
\hline T43 & $0.9510^{*}$ & 0.9351 & 0.9532 & 1.1404 & 0.9884 & 1.2994 & $0.8339 *$ & 0.6882 & 0.9278 & $1.2598^{*}$ & 1.0864 & 1.4391 & 0.9052 & 0 \\
\hline T44 & $1.4834^{*}$ & 1.4834 & 1.5152 & 1.4289* & 1.2470 & 1.6215 & 1.0382 & 0.8829 & 1.1603 & $1.3826^{*}$ & 1.1967 & 1.5238 & 1.0335 & 0 \\
\hline T45 & $1.0683^{*}$ & 1.0391 & 1.1116 & $1.1967 *$ & 1.0976 & 1.4000 & $0.8927^{*}$ & 0.7097 & 0.9721 & $1.1695^{*}$ & 1.0135 & 1.3332 & 1.0233 & 0 \\
\hline T46 & $0.6260^{*}$ & 0.5437 & 0.6585 & $0.6501^{*}$ & 0.5395 & 0.7458 & 0.9630 & 0.8090 & 1.0425 & $0.6449 *$ & 0.5589 & 0.7698 & 1.0080 & 0 \\
\hline T47 & $1.1466^{*}$ & 1.1392 & 1.1561 & $1.1785^{\star}$ & 1.0213 & 1.2863 & 0.9729 & 0.8757 & 1.0878 & 1.3013 & 0.8228 & 1.6144 & 0.9056 & 0 \\
\hline T48 & $1.1686 *$ & 1.1686 & 1.2030 & 1.1256 & 0.9891 & 1.2796 & 1.0382 & 0.8822 & 1.1611 & $1.1231^{*}$ & 1.0056 & 1.2529 & 1.0022 & 0 \\
\hline T49 & $1.1467^{*}$ & 1.1439 & 1.1527 & $1.1718^{*}$ & 1.0185 & 1.2842 & 0.9786 & 0.8748 & 1.0940 & $1.2223^{*}$ & 1.1014 & 1.3518 & $0.9587 *$ & 0 \\
\hline T50 & $1.2035^{*}$ & 1.1949 & 1.2144 & $1.1823^{*}$ & 1.0497 & 1.3176 & 1.0179 & 0.8835 & 1.1188 & $1.1940^{*}$ & 1.0689 & 1.3271 & 0.9902 & 0 \\
\hline T51 & 1.0897* & 1.0897 & 1.0921 & 1.0496 & 0.9091 & 1.1851 & 1.0382 & 0.8844 & 1.1610 & 1.0177 & 0.8706 & 1.1093 & 1.0314 & 0 \\
\hline T52 & 1.0203 & 0.9949 & 1.0719 & 1.0727 & 0.9290 & 1.1979 & 0.9511 & 0.8465 & 1.0648 & 1.1022 & 0.9878 & 1.2912 & $0.9732^{*}$ & 0 \\
\hline T53 & 1.0043 & 0.9810 & 1.0170 & 1.0000 & 0.8470 & 1.2009 & 1.0043 & 0.7546 & 1.1320 & 1.0000 & 0.7852 & 1.2393 & 1.0000 & 0 \\
\hline T54 & $1.0720^{*}$ & 1.0553 & 1.0869 & 1.0408 & 0.8522 & 1.1795 & 1.0300 & 0.8728 & 1.1838 & 1.0000 & 0.7869 & 1.1324 & 1.0408 & 0 \\
\hline T55 & $1.0354^{\star}$ & 1.0334 & 1.0377 & 1.0000 & 0.8303 & 1.1718 & 1.0354 & 0.8218 & 1.1839 & 1.0000 & 0.8158 & 1.1900 & 1.0000 & 0 \\
\hline T56 & 1.0056 & 0.9493 & 1.0261 & 0.9867 & 0.8314 & 1.1412 & 1.0191 & 0.8280 & 1.1416 & 0.9726 & 0.8096 & 1.1171 & 1.0145 & 0 \\
\hline T57 & $1.0662^{\star}$ & 1.0599 & 1.0912 & 1.0364 & 0.8623 & 1.2541 & 1.0288 & 0.7782 & 1.1848 & 1.0250 & 0.8430 & 1.2374 & 1.0111 & 0 \\
\hline T58 & $1.0331^{*}$ & 1.0267 & 1.0546 & 1.0440 & 0.9357 & 1.1537 & 0.9896 & 0.8846 & 1.0871 & 1.0629 & 0.9364 & 1.1944 & 0.9823 & 0 \\
\hline T59 & $0.9276 *$ & 0.8886 & 0.9703 & 1.0294 & 0.9490 & 1.1496 & $0.9011^{*}$ & 0.7782 & 0.9715 & 0.9702 & 0.8848 & 1.0372 & 1.0610 & 0 \\
\hline T60 & 1.0000 & 0.9893 & 1.0082 & $1.1655^{\star}$ & 1.1065 & 1.3524 & $0.8580^{*}$ & 0.6885 & 0.8983 & $1.1905^{*}$ & 1.1044 & 1.3153 & 0.9790 & 0 \\
\hline T61 & $1.0208^{*}$ & 1.0196 & 1.0449 & 0.9833 & 0.8601 & 1.1149 & 1.0382 & 0.8838 & 1.1596 & 0.9648 & 0.8286 & 1.0805 & 1.0192 & 0 \\
\hline T62 & 0.9712 & 0.9461 & 1.0211 & 0.9561 & 0.8137 & 1.1668 & 1.0158 & 0.7653 & 1.1618 & 0.9887 & 0.8100 & 1.2264 & 0.9670 & 0 \\
\hline T63 & $0.9324^{*}$ & 0.9104 & 0.9894 & 0.9045 & 0.7995 & 1.0678 & 1.0308 & 0.8280 & 1.1488 & 0.9299 & 0.7921 & 1.1116 & 0.9727 & 0 \\
\hline T64 & $1.2880^{*}$ & 1.2382 & 1.3867 & $1.2993^{*}$ & 1.1356 & 1.5050 & 0.9913 & 0.8420 & 1.1082 & $1.2558^{\star}$ & 1.0909 & 1.6696 & 1.0346 & 0 \\
\hline T65 & $1.2038^{*}$ & 1.1928 & 1.2085 & $1.1837^{*}$ & 1.0195 & 1.3467 & 1.0170 & 0.8498 & 1.1388 & $1.3757^{*}$ & 1.0055 & 1.6369 & 0.8605 & 0 \\
\hline T66 & $1.0217^{*}$ & 1.0190 & 1.0223 & 1.0048 & 0.8937 & 1.1227 & 1.0168 & 0.8807 & 1.1179 & 1.0095 & 0.9149 & 1.0929 & 0.9954 & 0 \\
\hline T67 & $0.6690 *$ & 0.6478 & 0.8509 & 1.0000 & 0.8898 & 1.4663 & $0.6690 *$ & 0.3988 & 0.7269 & 1.0000 & 0.7872 & 1.4131 & 1.0000 & 0 \\
\hline T68 & 0.9198 & 0.8833 & 1.0322 & 0.9647 & 0.9107 & 1.1500 & 0.9534 & 0.7789 & 1.0223 & 0.8882 & 0.8280 & 1.0086 & $1.0862^{*}$ & 1 \\
\hline T69 & $0.8213^{*}$ & 0.7837 & 0.8334 & $0.8566^{*}$ & 0.7357 & 0.9817 & 0.9587 & 0.7994 & 1.0704 & $0.8401^{*}$ & 0.7110 & 0.9582 & 1.0197 & 0 \\
\hline T70 & $1.1169 *$ & 1.0885 & 1.1661 & $1.2020^{*}$ & 1.1149 & 1.4157 & $0.9292^{\star}$ & 0.7536 & 0.9924 & $1.1614^{\star}$ & 1.0287 & 1.3807 & 1.0349 & 0 \\
\hline T71 & $1.0518^{*}$ & 1.0309 & 1.1003 & $1.1120^{*}$ & 1.0288 & 1.3043 & 0.9459 & 0.7732 & 1.0168 & $1.1412^{*}$ & 1.0606 & 1.3082 & 0.9745 & 0 \\
\hline T72 & $1.0351^{*}$ & 1.0282 & 1.0946 & 1.0559 & 0.9712 & 1.2470 & 0.9803 & 0.7999 & 1.0648 & 1.0274 & 0.8857 & 1.2104 & 1.0277 & 0 \\
\hline T73 & $1.0461^{*}$ & 1.0116 & 1.0770 & 1.0141 & 0.8834 & 1.1651 & 1.0315 & 0.8535 & 1.1427 & 1.0567 & 0.9340 & 1.2135 & 0.9597 & 0 \\
\hline T74 & 1.0016 & 0.9863 & 1.0159 & 1.0000 & 0.7360 & 1.2665 & 1.0016 & 0.6523 & 1.2040 & 1.0000 & 0.7171 & 1.2739 & 1.0000 & 0 \\
\hline T75 & 1.0119 & 0.9825 & 1.0251 & 0.9972 & 0.8451 & 1.1688 & 1.0148 & 0.8073 & 1.1401 & 0.9865 & 0.8146 & 1.1495 & 1.0109 & 0 \\
\hline T76 & $1.0354^{\star}$ & 1.0298 & 1.0504 & 1.0107 & 0.7908 & 1.2497 & 1.0244 & 0.7205 & 1.2117 & 1.0024 & 0.7622 & 1.2521 & 1.0083 & 0 \\
\hline T77 & $1.0595^{*}$ & 1.0595 & 1.0679 & 1.0205 & 0.8822 & 1.1785 & 1.0382 & 0.8515 & 1.1649 & 1.0000 & 0.7585 & 1.3720 & 1.0205 & 0 \\
\hline T78 & $1.0446^{*}$ & 1.0173 & 1.0690 & 1.0401 & 0.9236 & 1.1566 & 1.0043 & 0.8855 & 1.0986 & 0.9887 & 0.8023 & 1.1432 & 1.0520 & 0 \\
\hline $\begin{array}{l}\text { Geo- } \\
\text { mean }\end{array}$ & 1.0214 & - & - & 1.0763 & - & - & 0.9490 & - & - & 1.0667 & - & - & 1.0090 & \\
\hline
\end{tabular}

*It means that it is statistically significant at level $P<0.05$.

\section{Figures}



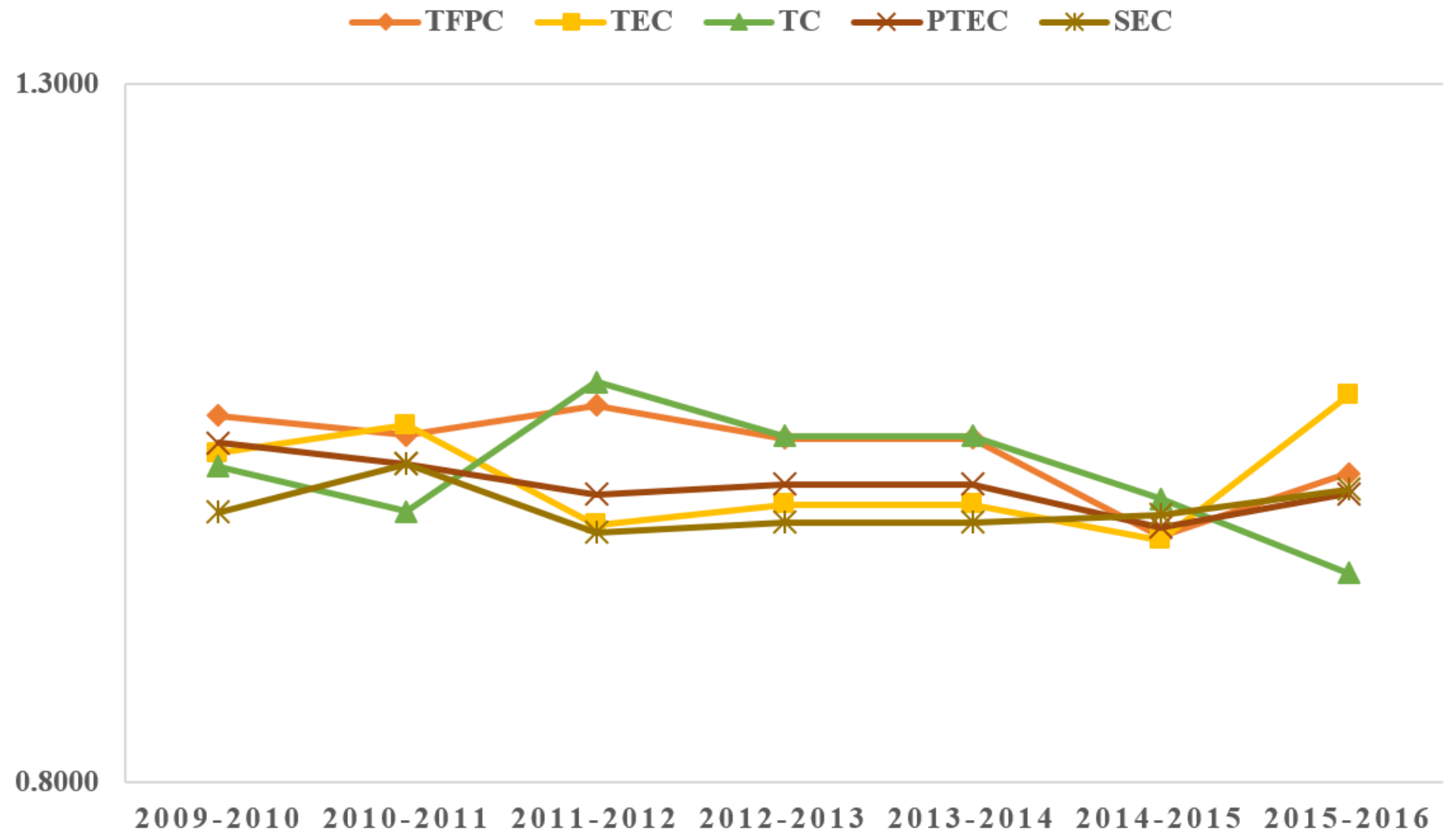

Figure 1

The trends of the TFPC and its decomposition index of the TCM hospitals from 2009 to 2016 